\title{
Automatic reconstruction of beam structures from 3D topology optimization results
}

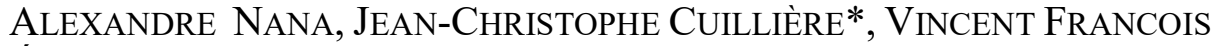 \\ Équipe de Recherche en Intégration Cao-Calcul, Université du Québec à Trois-Rivières, 3351 Boulevard \\ des Forges, Trois-Rivières, G9A 5H7, Québec, Canada. \\ ${ }^{*}$ Corresponding author \\ Jean-Christophe Cuillière \\ cuillier@uqtr.ca
}

\begin{abstract}
This paper presents a fully-automated reconstruction of beam-like CAD solid structures from 3D topology optimization (TO) results. Raw TO results are first processed to generate a triangulation that represents boundaries of the optimal shape derived. This triangulation is then smoothed and a curve skeletonization procedure is carried out to recover meaningful characteristics of this smoothed triangulation. The resulting skeleton, made with curvilinear geometry, is transformed into straight lines through a normalization process. These straight lines are used to generate a 3D beam structure. Thus, following these steps, a 3D beam structure is automatically derived from TO results. This 3D beam structure is meshed with beam finite elements and since TO non-design material is represented by 3D solid geometry, which is meshed using tetrahedron, the FEA beam structure needs to be rigidly connected with these tetrahedrons. Rigid connections between beam elements and 3D solid elements are ensured using specific FEA beam elements referred to as mini-beams. This results in a mixed-dimensional FEA model with beam and solid finite elements. Results obtained with this mixed-dimensional FEA model allow validating the beam structure obtained from TO results. Performance of the approach is demonstrated on several TO examples.
\end{abstract}

Keywords: topology optimization, geometry reconstruction, curve skeletons, beam structures, mixed-dimensional analysis.

\section{Introduction}

The aim of applying topology optimization (TO) in structural engineering [1] is to determine the optimal topology and geometry of a structural design which is submitted to specific constraints. It has become a very powerful tool in activities related to product design since it allows generating stiffer, lighter and more original shapes within a shorter time. The most popular TO approaches include homogenization methods [2], level sets methods [3], Evolutionary Structural Optimization (ESO) methods [4], and the Solid Isotropic Material with Penalization (SIMP) method [5]. Density-based approaches such as homogenization and SIMP algorithms result in an optimal distribution of material inside the space of an initially defined design domain. While composite microstructures are allowed as optimal solutions in homogenization-type methods, in the SIMP method, a penalization coefficient is introduced to reduce intermediate density values and force the optimal design into a discrete $0-1$ solution, where 1 represents fully solid material, and 0 represents the void. In level-set methods, a function describing the structural interface between solid material and void is used to parameterize geometry. Finally, ESO 
methods look for uniformly stressed designs by gradually removing inefficient material along the optimization process. Most of TO methods developed to this day are based on using finite element analysis (FEA) as numerical method along optimization iterations. To circumvent numerical instabilities that occur when using standard FEA, meshfree TO approaches have also been developed, especially when dealing with geometrically nonlinear structures [6].

Although most optimal designs resulting from TO consist of binary solid-void results, it remains that generating a CAD solid model for design optimization purposes from raw TO results still represents a major challenge. Indeed, optimal designs should be interpreted not only visually, but also quantitatively [5]. Moreover, the reconstructed CAD model should facilitate geometry modifications and reduce manufacturing constraints. In this context, manufacturability of the optimized designs provided by TO methods is a major concern. A very interesting survey and classification of manufacturing oriented topology optimization methods can be found in [7]. To avoid tedious manual interpretation and reconstruction operations, TO result reconstruction should be as automatic as possible to reduce time, limit designer intervention and facilitate the integration of TO methods into the overall design process. In this direction, this paper presents a new and fully-automated approach to reconstruct 3D CAD solid models from TO results. It is important to note that the approach presented in this paper is specifically intended for processing TO results that tend towards beam-like structures. From raw TO results, our method automatically generates CAD models composed with sets of straight beams. In this paper, TO results are obtained using the SIMP method but it is worth mentioning that the principles in which the proposed reconstruction approach are based can be applied to other TO schemes.

The paper is organised as follows. In the next section (section 2) previous work on interpreting 3D TO results is presented and discussed along with a brief review on curve skeletonization techniques. After presenting how TO results are generated in section 3, section 4 describes in detail the proposed reconstruction approach. Section 4 also introduces so-called mini-beams, which are standard FEA beam elements used to ensure rigid connection between beam and solid finite elements. In section 5, TO examples are used to illustrate the effectiveness of our approach. The paper ends with a conclusion about potential improvements of the approach and directions for further research on the subject.

\section{Related work}

Raw results provided by TO methods cannot be used as is for design purposes, and various techniques have been proposed to address the automatic conversion into CAD models of TO results. These methods can be categorized into two main strategies. The first strategy is based on iso-line (in 2D) and iso-surface (in 3D) reconstruction techniques. These methods use the relative density distribution, which means that they cannot be applied to TO approaches that are not based on relative density distributions. The second strategy involves black and white image interpretation methods.

With respect to the first strategy introduced just above, Youn et al. [8] extracted optimal shapes from density distributions provided by 2D TO simulations. In their approach, the shape of optimized models is extracted as an iso-line. This principle has been later extended to 3D models by Hsu et al. [9]. The basic idea of their work is to start the process by improving quality of TO results based on transforming intermediate densities into solid material or void. Then, 
representative cross sections are computed using iso-density contours that are represented as BSpline curves. A smoothed CAD representation is then obtained by sweeping through these cross sections. Koguchi et al. [10] applied a surface reconstruction algorithm on 3D TO results to obtain parametric CAD solid models using B-Spline surfaces. In their approach, iso-surfaces are first extracted from TO results, which is followed by feature detection, and biquartic surface splines reconstruction. Similarly, Tang et al. $[11,12]$ also used cross sections combined with Bspline surfaces extraction and geometric reconstruction to interpret $\mathrm{TO}$ results. However, because the reconstruction process is based on B-spline curves/surfaces, the optimized shape is complex and not easy to manufacture when using traditional machining processes. Moreover, most of these methods are semi or not automatic, since they rely on human intervention. According to the second strategy, Papalambros et al. $[13,14]$ have been among the first authors to use black and white image-interpreting techniques to convert TO results into CAD data. Their work is limited to 2D TO results and is based on B-Spline curves reconstruction. In their approach, the optimized 2D model is remeshed for further analysis and for subsequent sizing or shape optimization. Bremicker et al. [15] used image processing to interpret 2D truss structures. The medial axis transformation (MAT) is used to extract the skeleton of a truss-like structure generated by the TO process. The skeleton is later thinned and the result is reconstructed using bars. They are, to the best of our knowledge, the first to propose a skeleton extraction of truss and frame structures to interpret TO results. However, their implementation is limited to 2D optimization and requires designer interactions. Another effort to interpret TO result was presented by Lin and Chao [16]. They parameterized 2D TO results by using two shape characteristics, namely mean length and standard deviation from the centroid of each hole to its boundary. Values obtained for each interior hole are compared with characteristic values of seven predefined templates to interpret hole shapes. A shape optimization algorithm is finally applied after interpretation of the model. Since geometry reconstruction from TO results is only based on fitting predefined shapes, this reconstruction process is limited and very sensitive to the geometric complexity TO results. Later, Chou et al. [17] suggested to replace the shape characteristic value-based by polygons. Following the same idea, Larsen and Jensen [18] created smooth parametric CAD model from TO results. Each 3D feature of the model is obtained after fitting predefined 2D default templates to the optimization result, and sweeping the surface through the shape obtained. However, most of these methods are limited to 2D problems and they are not fully automated. In fact, the complete automation of these techniques is not yet achieved since operations such as the shape of templates and their number depends on the individual interpretation of the designer. Moreover, template approximation is not straightforward to extend to 3D TO problems, and templates used for geometric approximation are often inaccurate. This is why a shape optimization step is often necessary for refining and eventually restoring the design due to information loss.

The approach presented in this paper focusses on reconstructing CAD solid models from TO results that tend to $3 \mathrm{D}$ beam-like structures without any user intervention. The reconstruction process is fully automatic, and results in CAD models of beam structures with high manufacturability potential and that can be easily modified and manipulated for further editing in a CAD system. Since this work focuses on beam-like structures, automatic skeletonization methods are of a great interest [15]. Moreover, as mentioned in [19], processing and analysing the skeleton of a 3D shape can be an appropriate solution to assess its topology. Indeed, the skeletonization of a 3D model supplies a simplified and compact representation that is locally equidistant to points on the boundary of the model $[19,20]$. Automatic skeletonization 
techniques arouse a growing interest with recent advances in $3 \mathrm{D}$ acquisition devices and computer graphics. The main interest of these techniques is that they intuitively capture meaningful information (e.g. symmetry, adjacency, local complexity, number of branches and local width) of the topology of a model. A large number of methods can be found in the literature for skeletonizing 2D models, which is not the case for the skeletonization of 3D models. In general, the skeleton of a 3D shape can be a surface skeleton, such as the medial axis [21, 22], or a curve skeleton $[23,24]$. In the medial axis extraction, a medial function is used to assign high weights to skeletal points, and small weights to non-skeletal points. Then, the associated distance from skeletal points to the closest boundary is computed. This is achieved by using algorithms

such as distance transform, potential functions, thinning erosion and Voronoï diagrams. Even if effective, the medial axis is highly sensitive to small perturbations on boundaries of the shape, difficult and expensive to extract in 3D [25], and hard to store and manipulate [24]. Adding to this the fact that, in this work, we focus on beam-like structures, our attention is attracted by curve skeletons. The curve skeleton of an object can be defined as its thinned curvilinear representation [23]. It has been applied in many domains that include computer graphics, 3D animation, and medical visualization because of its intuitive description of the topological characteristics of a model [26-28]. Although well documented in the literature, curve skeletonization algorithms have been mostly implemented in a context of image processing and object recognition where the topology of the model was known a priori, but lost during the data acquisition process. In this study, curve skeletonization is used to simplify the topology of the optimized 3D shape obtained and to reconstruct manufacturable structures from beam-like TO solutions.

\section{Generating structural TO results}

Beam-like structures are likely to be obtained in TO results when using refined meshes and small volume fractions $[15,29]$. In this paper, structural TO results are obtained using the SIMP method in 3D. It is a choice among others since other TO methods could have been considered. Indeed, the methodology proposed in this paper operates on any $3 \mathrm{D}$ optimized shape with a boundary that can be represented as a triangulation or even as a point cloud.

The SIMP method consists in minimizing the compliance $\tilde{C}$ of a 3D model subjected to a volume fraction constraint. This volume fraction represents the percentage of the initial design material that is kept along SIMP iterations. The method uses a finite element calculation at each iteration and it converges when the relative difference in global compliance between two successive iterations is less than a given threshold $\left(\Delta_{c o n v}=0,5 \%\right.$ in this study). Finite element calculations are used to update a material relative density distribution $\rho(x, y, z)$ which is constant within each element of the mesh. The relative density $\rho(x, y, z)\left(\rho_{e}\right.$ in each finite element $\left.e\right)$ is related to the distribution of a virtual elastic modulus according to the penalization law:

$$
\tilde{E}(x, y, z)=E \cdot(\rho(x, y, z))^{p}
$$

Where $p$ is a penalization coefficient ( $p=3$ is considered in this work).

In the following equations, all parameters that are affected by $\rho(x, y, z)$ are noted using a $\sim$ The global stiffness matrix $[\widetilde{K}]$ derived from local matrices $\left[\widetilde{K}_{e}\right]$ for $N$ finite elements $e$ is given by: 


$$
[\widetilde{K}]=\sum_{e=1}^{N}\left[\widetilde{K}_{e}\right]=\sum_{e=1}^{N}\left(\rho_{e}(x, y, z)\right)^{p} \cdot\left[K_{e}\right]
$$

A classical formulation of the SIMP topology optimization method is:

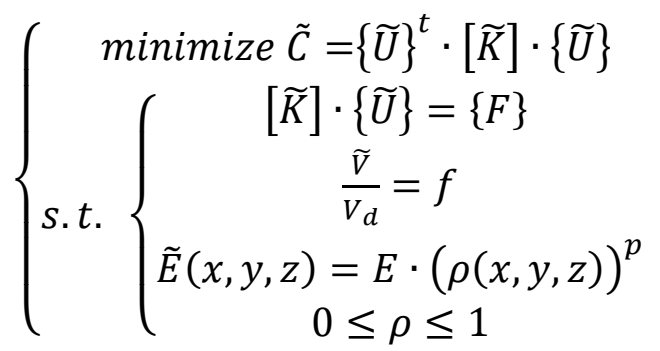

Where $\tilde{C},\{\widetilde{U}\}$ and $\{F\}$ are respectively the global compliance of the whole domain, the global displacement vector and the global load vector. $f$ is the volume fraction, which is a constraint imposed on the TO process. It is calculated as the ratio of $\tilde{V}$, the prescribed design material volume that is kept constant along SIMP iterations, and $V_{d}$, the actual total design material volume. Practically, global compliance is computed as $\tilde{C}=2 \cdot \widetilde{W}$, where $\widetilde{W}$ is the total strain energy inside the whole model as obtained in the FEA calculation associated with the last SIMP iteration.

Once convergence is achieved, the raw SIMP result obtained is the final relative density distribution $\rho(x, y, z)$. The rough shape of the optimized model derived from this relative density distribution consists, as explained in [30], in a set of finite elements with a density value that is over a user-specified threshold $\rho_{t h}$. Details on the SIMP method and its implementation can be found in [5, 31], and improvements in the description of the optimal shape boundary through adaptive TO are addressed in $[32,33]$. Our implementation of the SIMP method is based on FEA with linear tetrahedral elements and Code_Aster ${ }^{\mathrm{TM}}$ [34] is used as FEA solver. Note that all figures shown in this paper are generated using $\mathrm{Gmsh}^{\mathrm{TM}}$ [35].

An initial sample part (overall dimensions are $304,8 \mathrm{~mm} \times 355,6 \mathrm{~mm} \times 762 \mathrm{~mm}$ ) with boundary conditions and loads $\left(17,2 \mathrm{kN} / \mathrm{m}^{2}\right.$ and $8,0 \mathrm{kN} / \mathrm{m}^{2}$ in respectively $Y$ and $Z$ directions) is illustrated in Figure 1a. Figure 1b shows the 3D uniform tetrahedral mesh (mesh size $d_{g}=$ $13 \mathrm{~mm}$ ) of this sample part with design material (material that will be optimized) in blue and non-design material (that is kept intact along the optimization) in red. Young's modulus is $E=$ $69 \mathrm{GPa}$, Poisson's ratio is $v=0,33$, and the volume fraction is $f=8 \%$ (thus targeted design material volume is $\tilde{V}=4,72 \cdot 10^{-3} \mathrm{~m}^{3}$ ). The optimal density distribution obtained at convergence is illustrated in Figure 1c. Compliance reached at convergence is $\tilde{C}=9,6$. $10^{-3}$ Joules, which corresponds to an optimized material volume $\tilde{V}_{o p}=4,71 \cdot 10^{-3} \mathrm{~m}^{3} . \tilde{V}$ is the volume target before SIMP optimization while $\tilde{V}_{o p}$ is the optimized material volume obtained after SIMP optimization.

Figure 2a illustrates the 3D shape obtained from the relative density distribution at convergence shown in Figure 1c. This optimized shape is based on keeping elements for which relative density $\rho \geq \rho_{t h}=0,45$. The threshold value is based on two aspects: the target volume derived from volume fraction and material continuity. Indeed, while reconstructing TO results, continuity in the distribution of the optimized material is of great importance. The compliance of a 
continuous structure is lower (the structure is stiffer) than that of a discontinuous structure [9]. Thus, in [9] it is suggested that the extraction threshold value $\rho_{t h}$ should be chosen with respect to continuity of the optimized design. Consequently, in our approach, $\rho_{t h}$ is chosen so that the target volume is achieved and that model continuity is respected.
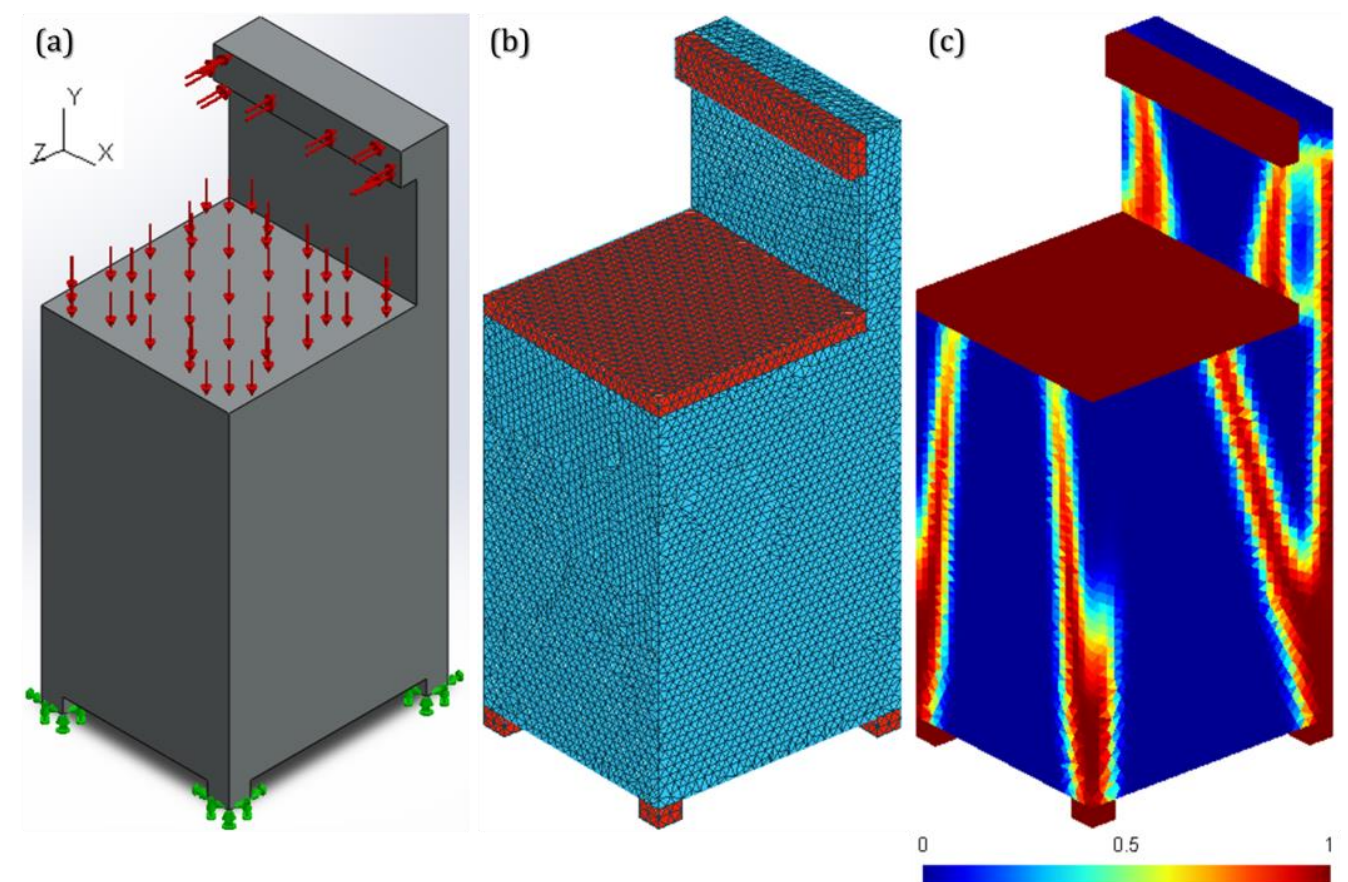

Figure 1 : Chair (a) geometry, loads and boundary conditions (b) design (in blue) and non-design (in red) material using a uniform mesh size $\left(d_{g}=13 \mathrm{~mm}\right)(\mathrm{c})$ relative density distribution after convergence of SIMP optimization.

Figure $2 b$ illustrates the rough optimal shape extracted, as a triangulation, from the result shown in Figure 2a where the non-design domain is in yellow, and the optimized design domain is in grey. Figure 2c shows the resulting optimized shape only, where non-design material has been removed. In this figure, triangles that are at the interface between non-design and design material are in yellow. The volume of the optimized design domain (shown in Figure 2c) is $V_{s h}=4,8$. $10^{-3} \mathrm{~m}^{3}$. This volume also represents the $3 \mathrm{D}$ shape that has to be reconstructed through the next steps, since non-design material remains unaffected by the optimization process.

As outlined earlier and as illustrated in Figure $2 \mathrm{~b}$ and Figure 2c, the outcome of TO requires substantial post-optimization processing before being able to be used in the CAD process. If CAD model reconstruction from TO results is fully automated, integration of TO in the overall product development process is optimized. Thus, a new approach to the automatic reconstruction of 3D solid models from TO results is proposed and validated through a set of illustrative case tests. As mentioned before, the proposed approach is based on using curve skeletonization methods. 

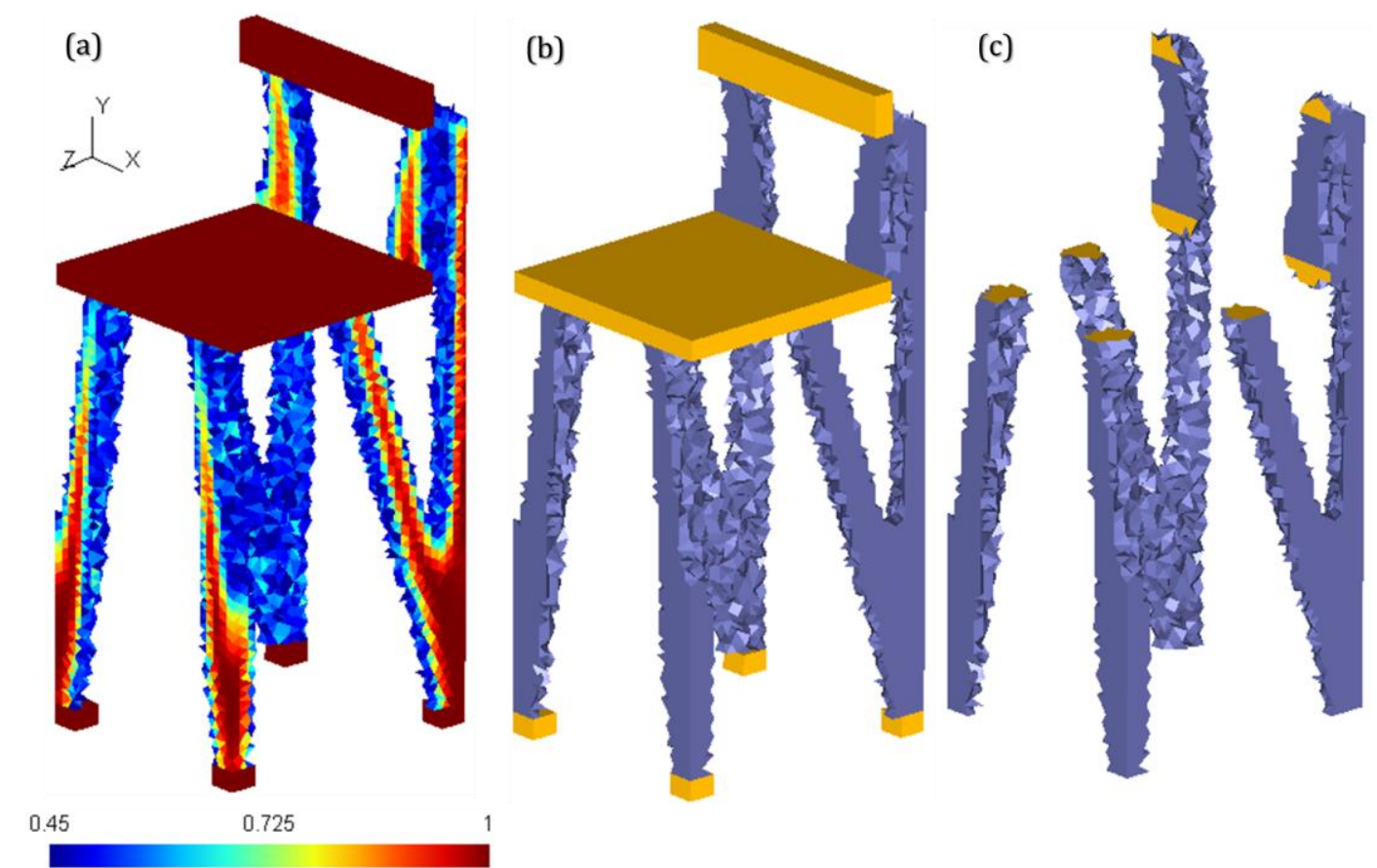

Figure 2 : Chair (a) raw optimized shape using $\rho_{t h}=0,45$, (b) rough optimal design as a triangulation with the optimized shape (in grey) and non-design material (in yellow) (c) rough optimized shape to be reconstructed (non-design material excluded).

\section{Automatic reconstruction of 3D beam-like structures from TO results}

Integrating TO into an automated structural design procedure represents a highly promising outcome of using TO for computer aided design. However, it also represents a major challenge since, as introduced just above, optimized results cannot be used as is for design purposes. This section details the proposed reconstruction approach and starts with an overview of our method.

\subsection{Overview of the approach}

The input of our reconstruction approach is the boundary triangulation that represents the optimized shape derived from TO result as illustrated in Figure 2b. A workflow of our approach is proposed in Figure 3. The reconstruction process starts with smoothing this boundary triangulation to remove the noise that is present on the rough boundary of the optimized model. Since, as illustrated in Figure $2 b$, the rough boundary of the optimized model is affected by high amplitude noise, this requires using very specific smoothing procedures. Noise smoothing is followed by curve skeletonization and by the computation of cross-section parameters of the skeleton segments. These segments are then transformed into straight beams through a normalization process which takes place once the skeleton and associated cross section parameters are generated. These segments are meshed with beam finite elements and are then connected rigidly with non-design material. Since non-design material is represented by 3D solid geometry and meshed with solid finite elements (tetrahedrons in our case) a specific connection strategy needs to be used to rigidly connect $3 \mathrm{D}$ beam elements with tetrahedrons. The connection between the mesh of the normalized skeleton (the reconstructed optimized design material) and 
3D solid elements (non-design material) is ensured using conventional beam finite elements referred to as mini-beams. As shown in Figure 3, the reconstructed model is finally validated with FEA simulations. This validation aims at assessing performance of the 3D structure that has been automatically reconstructed. It is performed using a mixed-dimensional FEA model made of beam and solid tetrahedral finite elements, which is automatically generated from reconstruction results.

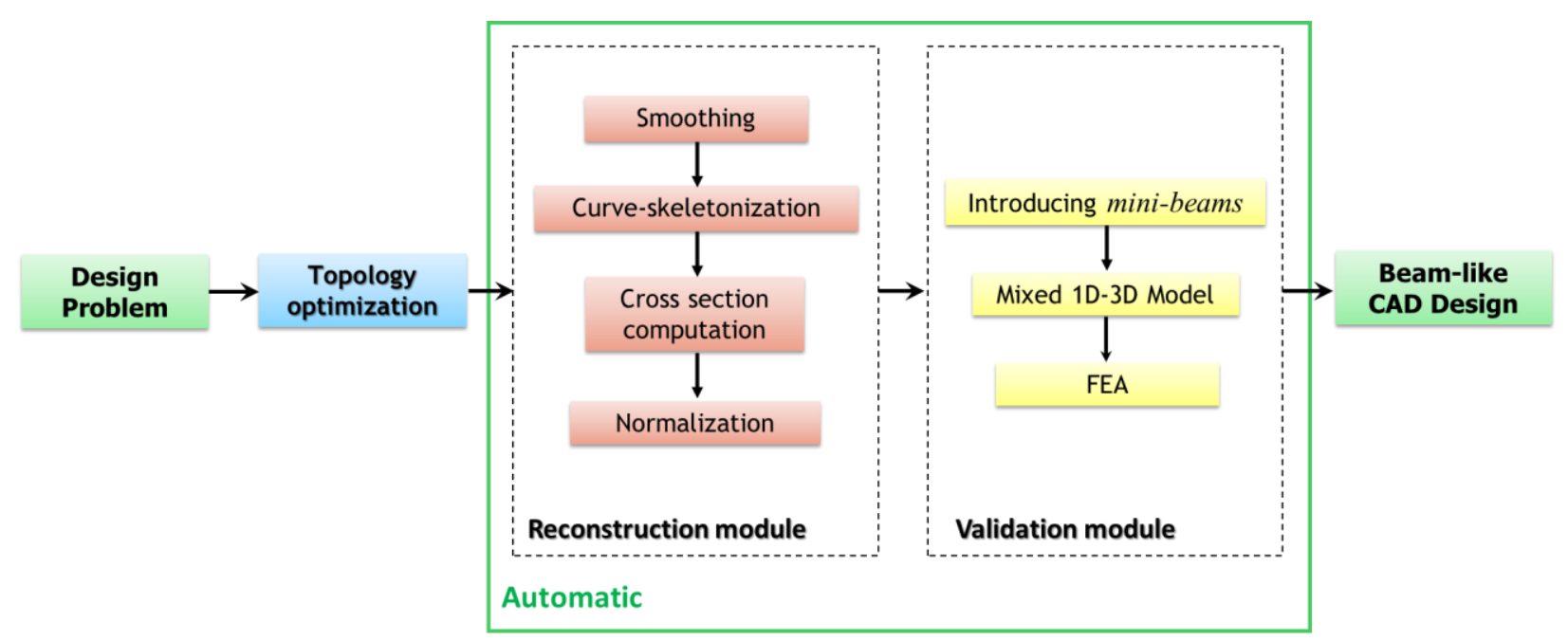

Figure 3 : Flow chart of the proposed TO data reconstruction approach.

In the proposed approach, non-design material remains unchanged along the overall reconstruction procedure. Non-design material typically represents functional zones of the model that are often associated with boundary conditions and loads. Moreover, this non-design material is not modified during the optimization process and hence does not require reconstruction.

The approach proposed in this workflow may seem similar to that in [15]. However, the work presented in [15] is limited to 2D cases and does not feature any validation of reconstructed structures. Furthermore, compared with other TO results reconstruction methods such as imagebased interpretation strategies $[16,17]$ or iso-density-based interpretation $[9,10]$, the proposed algorithm is fully-automatic, is applied to 3D problems, can be applied regardless the optimization method utilized, and is designed for the reconstruction of 3D TO results that contain beam-like structures. The different steps of the workflow presented in Figure 3, for both reconstruction and validation modules, are described in the next sections.

\subsection{Reconstruction module}

\subsubsection{Boundary smoothing}

As presented in Figure 3, in the objective of automatically reconstructing CAD solid models from TO results, the first step is to smooth boundaries of optimized domains generated by TO. These boundaries are extracted from the rough optimized shape as triangulations. Smoothing triangulations of arbitrary topology has been studied for decades in different fields such as computer aided design, computer vision, 3D animation, 3D scanners, medical imaging, etc. 
Indeed, in many applications, input triangulations feature noisy data (such as noisy point clouds coordinates acquired from 3D scanners) and this noisy data requires smoothing so that the output is a smoothed mesh, where high frequencies are filtered out. Many triangulation smoothing methods are available in literature. Among these methods, some target preserving features while removing noise from the mesh [36], and others are more focussed on quality of smoothed triangulation, such as Laplacian-based methods [37]. Smoothing methods are used in many fields (CAD, FEA, computer graphics, medical imaging, animation, etc.). However, none of these methods can successfully process very noisy triangulations provided by TO processes (such as the triangulation shown in Figure 2b). Indeed, noise on the surface of a TO result is not a mathematical function injected in the model, or a noise resulting from data acquisition. Moreover, its amplitude is very high, which makes it difficult to smooth efficiently. Ideally a good smoothing procedure for processing TO results should produce smooth surfaces, preserve some of the features generated by TO and meet TO volume targets. Designing a smoothing algorithm that is well suited for processing TO results remains a challenge that needs to be addressed. We achieved a satisfactory solution by combining two smoothing methods $[37,38]$ to have high quality in the representation of the optimal shape surface while preserving volume and shape of the optimized material. It is worth noting that, in this work, the main objective of smoothing is to facilitate curve skeletonization and the computation of beam cross section parameters, as described in the next section. Figure 4a illustrates the results obtained after smoothing the boundaries of results shown in Figure $2 b$.
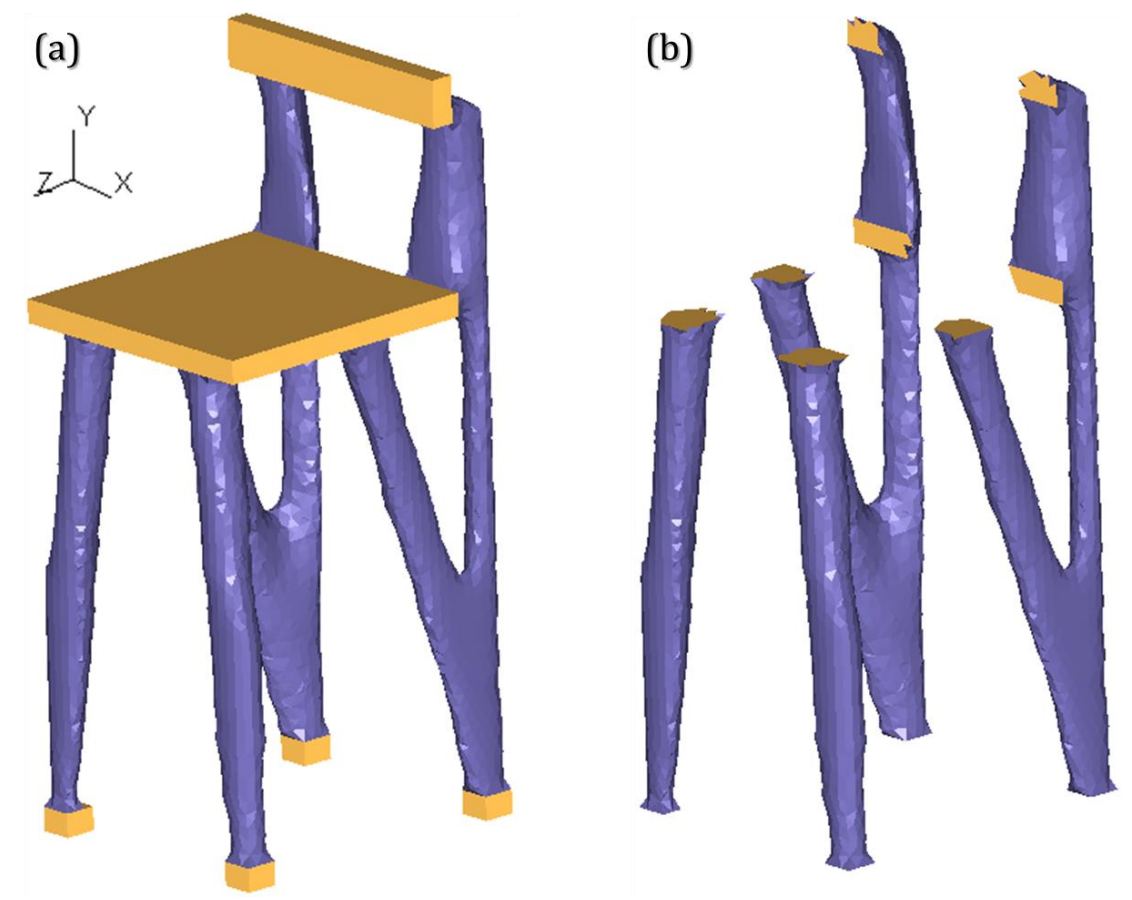

Figure 4 : Chair (a) smoothed optimized shape (in grey) with non-design material (in yellow) (b) the smoothed optimized shape to be reconstructed (non-design material excluded).

If smoothed results shown in Figure 4a are compared with the raw TO shape shown in Figure $2 b$, it is obvious that high frequencies have been filtered out and that the triangulation representing the smoothed shape is more regular. Since no reconstruction is needed for non-design material, 
smoothing is only applied to the boundaries of the optimized design material and not to boundaries of non-design material. Figure $4 \mathrm{~b}$ presents the smoothed optimal shape (non-design material excluded). The volumes of the optimized design material before and after smoothing are respectively $V_{s h}=4,84 \cdot 10^{-3} \mathrm{~m}^{3}$ and $V_{s m}=4,83 \cdot 10^{-3} \mathrm{~m}^{3}$.

\subsubsection{Curve skeletonization}

The objective of this work is to process beam-like 3D TO results in order to automatically generate $3 \mathrm{D}$ beam structures. This is performed using a curve skeletonization method. Compared to other skeletonization techniques such as the medial axis [21,28], curve skeletonization has been chosen in this work since it is a powerful shape abstraction tool that is particularly well fitted for processing beam-like shapes [24]. Using curve skeletonization techniques simplifies the reconstruction of beam-like structures and makes the proposed approach more easily extendable to any other TO method and more easily automated. The skeletonization method used in this work is based on a mesh-contraction Laplacian-based algorithm presented in [27, 39]. This method is fully automated and has been initially designed for retrieving 3D models from scanning devices point clouds. The main principle on which it is based is to iteratively contract the model's boundary until it reaches zero-volume. The contracted shape is then converted into a curvilinear skeleton. This curve skeleton is obtained by sub-sampling the contracted skeletal point cloud and by connecting sub-samples with their 1-ring neighbors. A segment collapse thinning process is finally applied to obtain the final curve skeleton. Figure 5 shows the curve skeleton obtained from the smoothed optimized shape presented in Figure 4a.
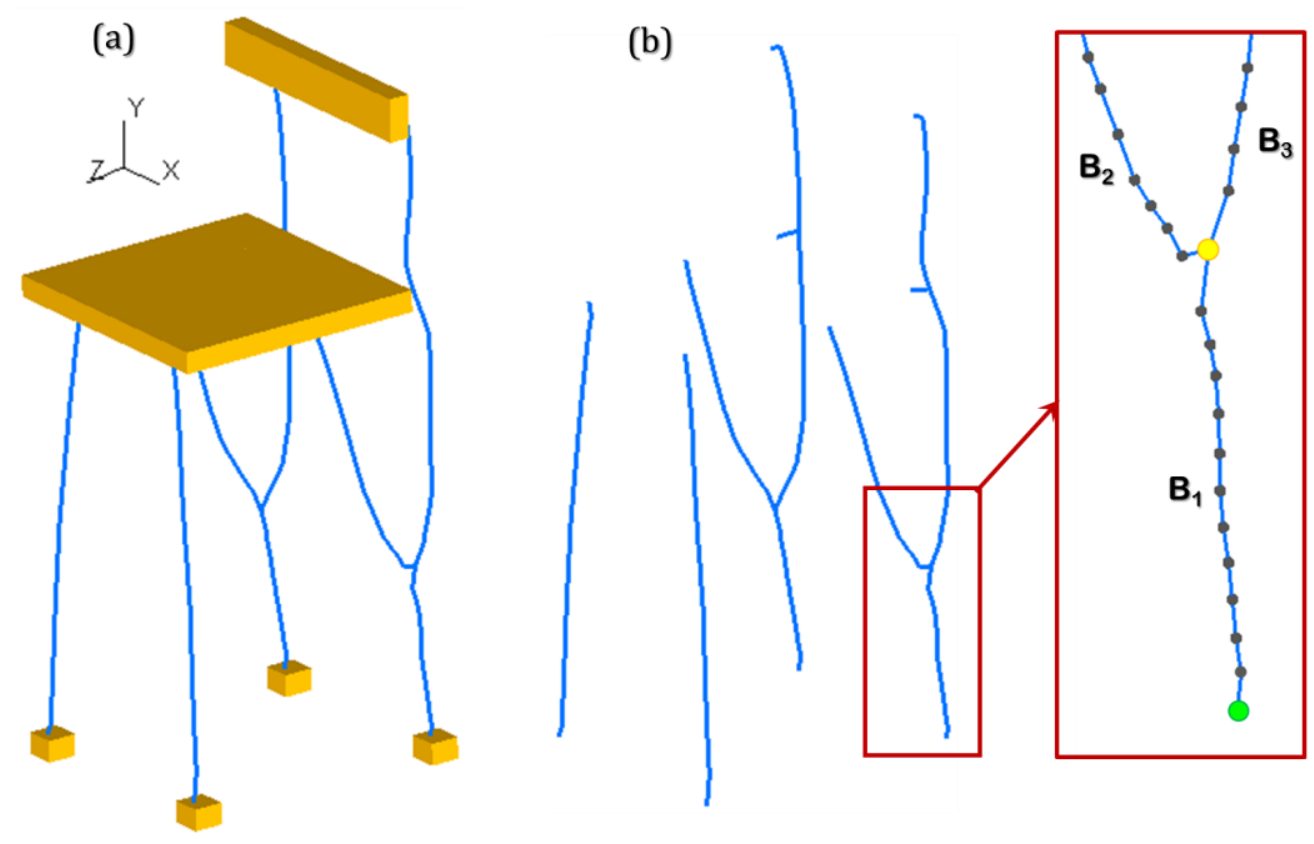

Figure 5 : Chair (a) curve skeleton with design material as curves (in blue) and non-design material (in yellow) (b) curve skeleton (c) distribution of beam radius along each beam of the curve skeleton. 
It also shows that, if compared with the smoothed TO shape, topology of the optimized structure is well captured using such a curve skeletonization process. In Figure 5a, non-design material (in yellow) is represented by solid tetrahedral finite elements while design material is represented by wireframe elements (in blue). Figure $5 \mathrm{~b}$ represents wireframe elements only, which means with non-design material excluded. The curve skeleton consists of 184 segments (in blue in the zoom), 10 end points (like the green point in the enlarged view), 4 junction points (like the yellow point in the enlarged view), and 10 branches (like branch $\mathrm{B}_{1}$ in the enlarged view which is composed of 11 branch points in grey and 12 segments). The first and last points of a branch correspond to junction or end points. This information will be later used in the normalization process to transform each branch into a straight beam.

\subsubsection{Computation of cross section parameters}

Once a curve skeleton is extracted, each beam segment of this skeleton is approximated as a beam with circular cross section. This means that only one parameter, the section radius, needs to be computed. The radius of each beam cross section is calculated by computing, along each curvilinear segment of the skeleton, the mean local Euclidian distance between the segment and the smoothed triangulation. Actually, as shown in Figure 6a, considering a beam segment (in blue), the two end points of a skeleton segment (in pink) are used to select nodes of the smoothed triangulation that will be considered for the calculation of the cross section radius. Selected nodes are in black in the figure while discarded nodes are in red. Each selected node $\mathrm{P}_{i}$, is orthogonally projected onto the beam segment as $\mathrm{Q}_{i}$. The Euclidian distance $\mathrm{P}_{i} \mathrm{Q}_{i}$, is then calculated, and stored as a local radius $R_{i}$. Finally, the beam cross section radius is computed as the average value of local radii $R_{i}$. The result obtained after this radius computation is shown in Figure $6 \mathrm{~b}$.

(a)

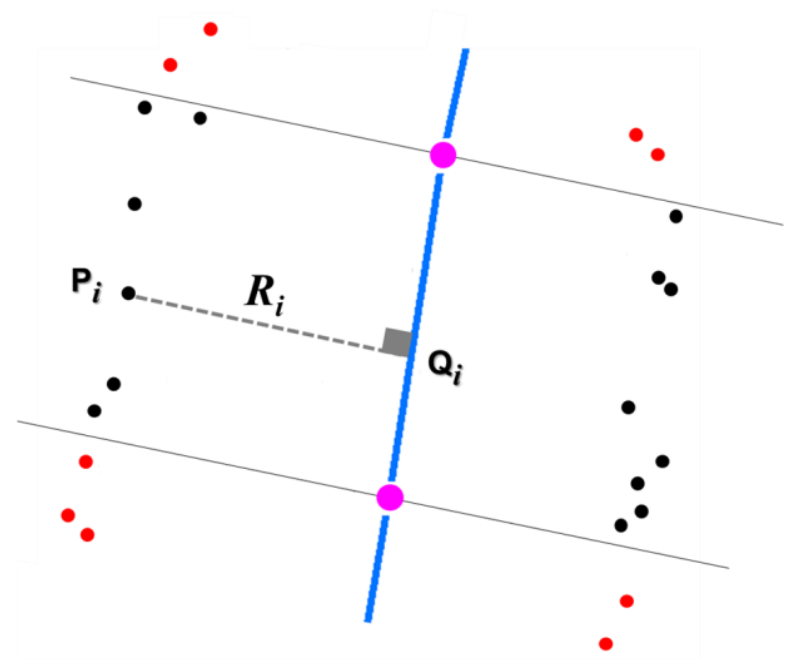

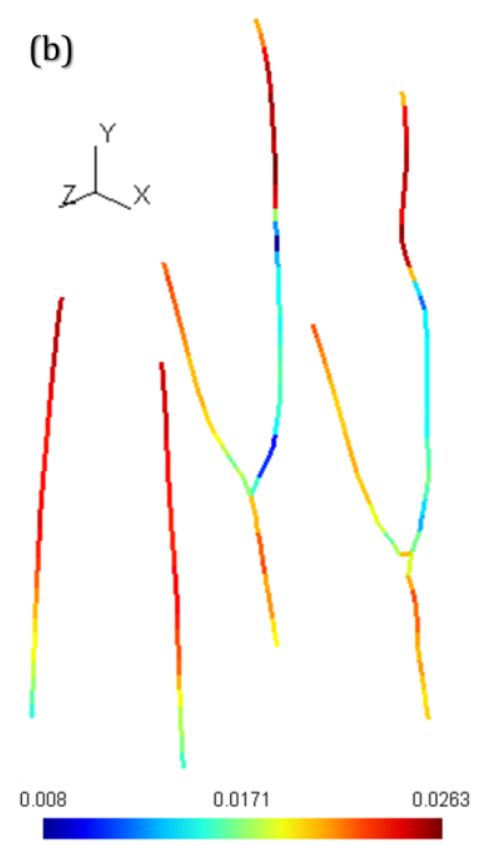

Figure 6 : (a) Illustration of the cross section calculation process and (b) the resulting distribution of beam radius along each beam of curve skeleton for the chair (in $m$ ). 


\subsubsection{Normalization of the skeleton}

As introduced in section 4.1, normalization is a tuning process that simplifies the curve skeleton into a set of straight beams with uniform circular sections. 3D designs produced by the optimization process usually feature shapes and topologies that increase the manufacturing cost when feasible [12]. In most cases, it is very difficult to transform raw TO results into 3D solid models that can be easily manufactured without a significant loss of the optimization benefits. Moreover, since manufacturability is one of the main factors that influence the final cost of a design, it is of major importance since early design stages [9]. Being able to reduce manufacturing difficulties (especially when using traditional manufacturing processes) without losing the benefits of TO is among main objectives of this normalization step. Figure 7 shows the result obtained after normalization of the result shown in Figure 5. Each branch of the skeleton, previously composed with a curvilinear beam, is transformed into a straight beam with a uniform cross section along its length. The cross section of each of these straight beams is calculated as the average of cross section radii of beam segments along the curvilinear beam of a branch. As an example, the 12 beam segments of branch $\mathrm{B}_{1}$ (see the zoom in Figure $5 \mathrm{~b}$ ) are transformed into one straight beam as shown in the enlarged view in Figure $7 \mathrm{~b}$. Radius of this new straight beam is calculated as the average value of cross section radii of the 12 beam segments. Thus, 10 straight beams are generated from the 10 branches of the curve skeleton. Figure $7 \mathrm{a}$ and Figure $7 \mathrm{~b}$ respectively correspond to Figure $5 \mathrm{a}$ and Figure $5 \mathrm{~b}$ after normalization. Radii associated with each branch after normalization are shown in Figure 8a. As explained just above, cross-section radius along each straight branch is constant (see Figure 8a), which was not the case along curvilinear branches before normalization (see Figure 6b). The reconstructed CAD model is shown in Figure 8b. As detailed in the next section and as illustrated in Figure 7a, a particular attention must be paid on connections between wireframe elements (design geometry) and solid elements (non-design geometry).

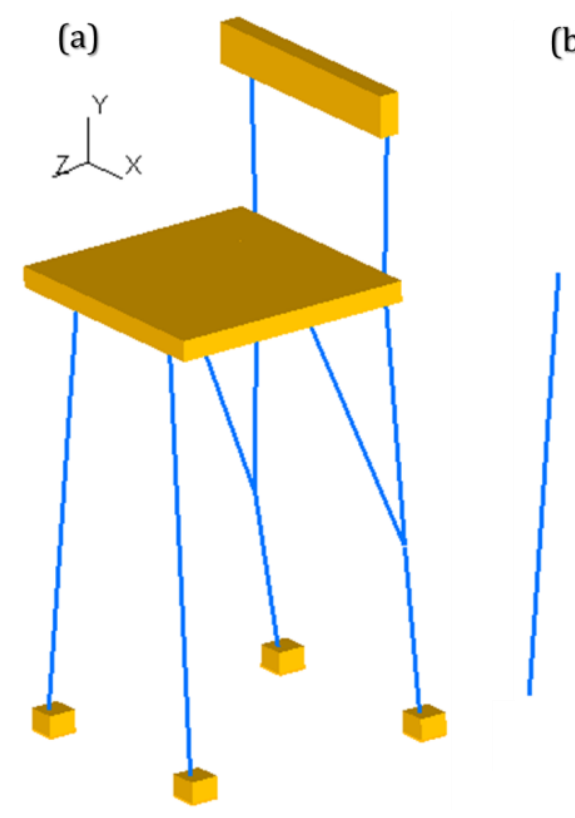

(b)

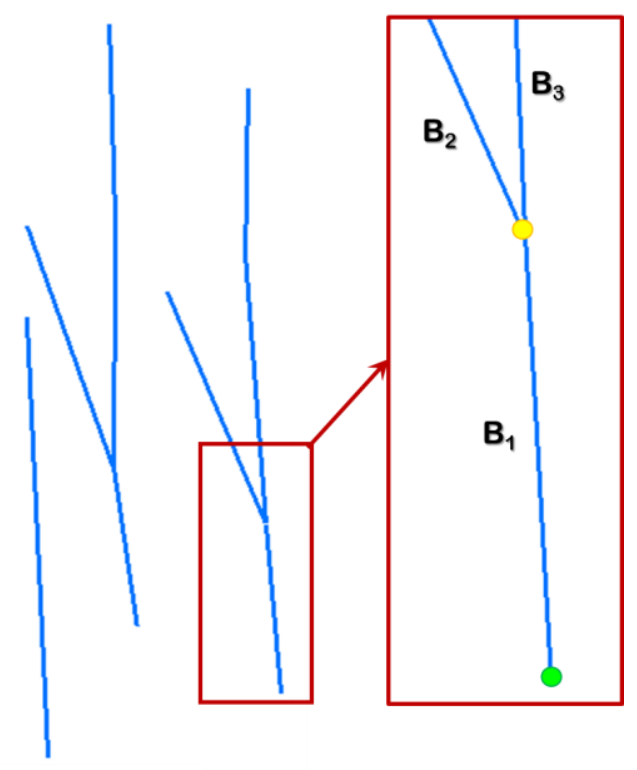

Figure 7 : Chair (a) normalized skeleton with the optimized structure (in blue) and non-design material (in yellow) (b) normalized skeleton (non-design excluded). 

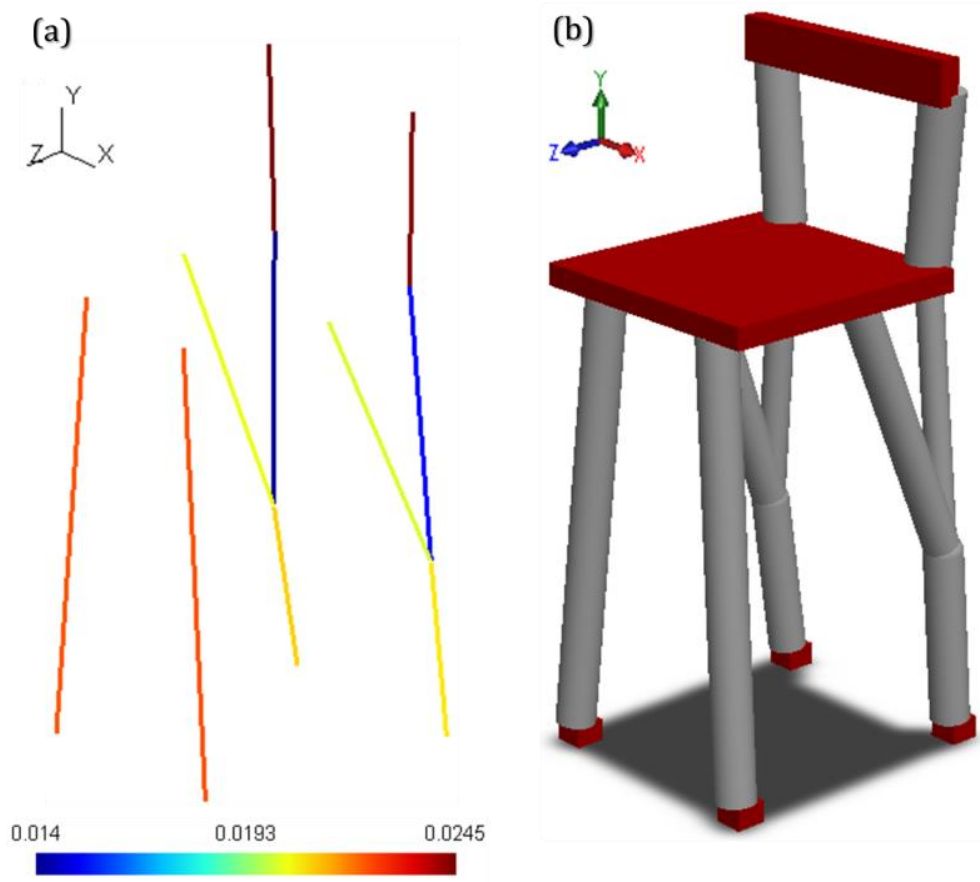

Figure 8 : (a) Radii of normalized skeleton beams (in $m$ ) and (b) the reconstructed beam-like CAD chair model.

\section{$4.3 \quad$ Validation module}

Validating the reconstructed model is the second module in our methodology. It can be performed by applying, to the mixed-dimensional FEA finite element model generated in the previous step, the same boundary conditions and loads as those applied in the TO process itself (shown in Figure 1a for our illustration example). Thus, strength of the optimized beam structure model generated with our approach from TO results can be assessed.

\subsubsection{Connection between beam and solid finite elements}

The connection between the normalized skeleton and non-design material is the last step of our reconstruction process. Indeed, once we meshed the normalized skeleton (with beam finite elements) and non-design material (with linear tetrahedrons), some of the beam elements of the normalized skeleton need to be rigidly connected with tetrahedrons of non-design material. This requires specific processing since these finite elements do not feature the same number of degrees of freedom (DOF) per node. Indeed, beam elements usually feature 6 DOF while solid elements used in linear elasticity only feature 3 DOF. Various solutions have been proposed for handling this type of inconsistency between degrees of freedom of beam and solid elements at solid-wireframe interfaces. In our case, as described with details in [40], it is solved using socalled mini-beams. As explained in [40], these mini-beams ensure a rigid connection between beam and solid finite elements 3D in the final FEA model. This solution has proven to be both simple and effective since these mini-beams are standard FEA beam elements used for connection purposes, which means that it does not require using neither specific finite elements nor additional constraints [40]. The rigid connection between beam and solid elements is made at each interface by connecting straight beams to a node on the surface of the 3D mesh, which is 
connected to a set of mini-beams. Figure 9a illustrates a connection with such mini-beams for the result shown in Figure 7a. In the figure, mini-beams (in blue) are added on all the adjacent segments of the connecting node localized on the surface of the $3 \mathrm{D}$ mesh (in yellow). This ensures a rigid connection instead of a ball joint connection in the FEA model. One of the main advantages of this approach is that, since it uses standard finite elements only, it can be used with any FEA package. The result obtained is a mixed-dimensional FEA finite element model that will be used to validate the optimal structure design obtained throughout the process.

\subsubsection{Mixed-dimensional FEA model}

The mixed-dimensional FEA model used for validation is obtained by meshing beams with standard beam elements and meshing 3D non-design geometry with tetrahedral elements. Practically, only straight beams need to be meshed at this stage, since non-design geometry was already meshed for TO. Thus, we keep the same mesh for non-design geometry and uniformly mesh wireframe geometry using the same element size. Accordingly, straight beams are meshed using the same uniform mesh size as the initial tetrahedral mesh used for TO $\left(d_{g}=13 \mathrm{~mm}\right.$, see Figure 1b), as illustrated in Figure 9b.
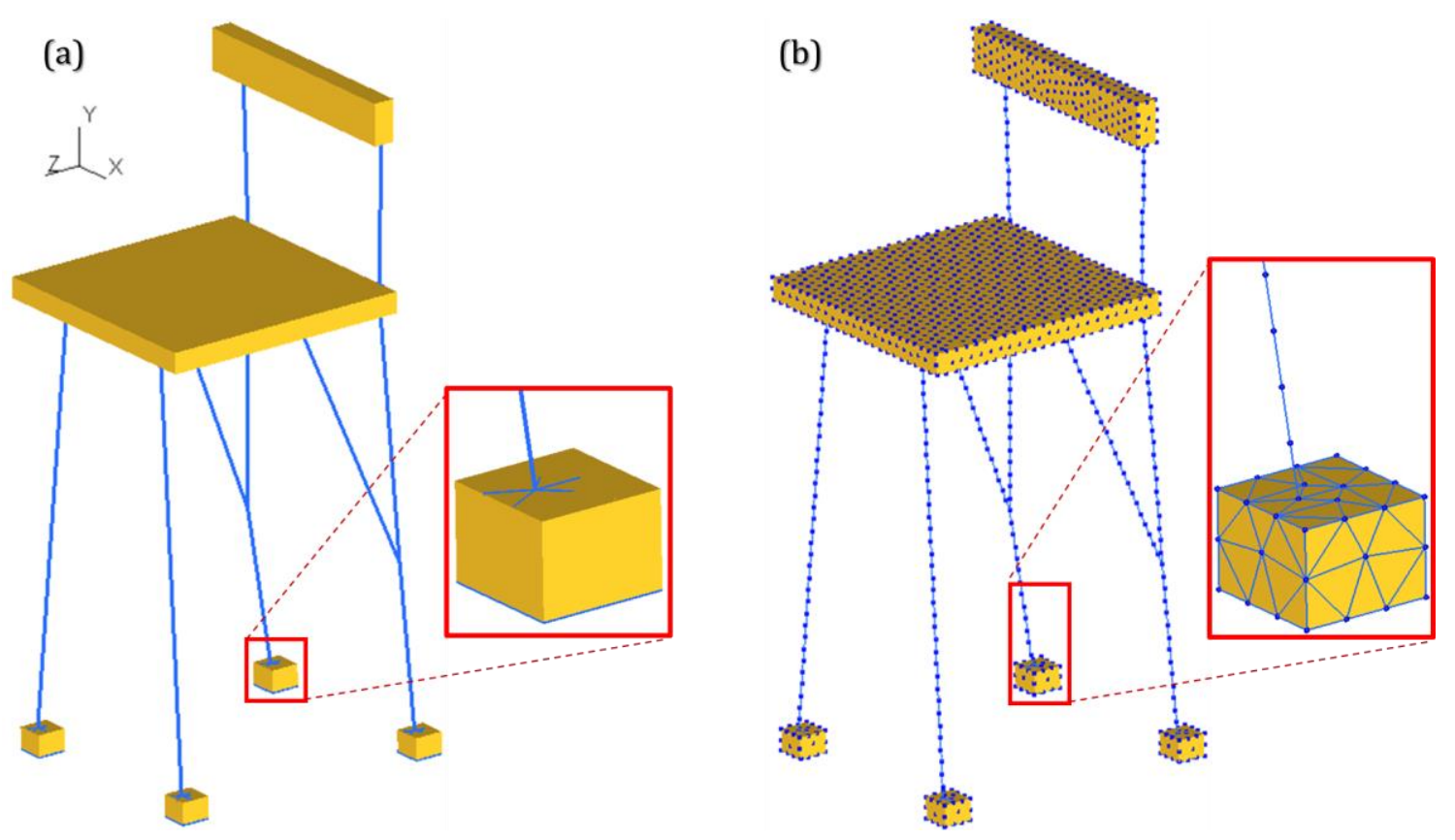

Figure 9 : Mixed-dimensional FEA model of the chair (a) illustration of mini-beams (b) mesh.

\subsubsection{FEA validation of the optimal structure obtained}

Figure 10 shows results obtained with the mixed-dimensional FEA model introduced just above. Figure 10a illustrates the distribution of the norm of displacement vector (in $\mathrm{mm}$ ) while Figure $10 \mathrm{~b}$ illustrates the distribution of elementary strain energy (in Joules). From this elementary strain energy distribution, we can compute the strain energy of the whole reconstructed structure $W_{r}$, computed as the sum of the energy in each beam and tetrahedral element (thus including non-design material). For this example $W_{r}=4,40 \cdot 10^{-3}$ Joules, which can be related to an equivalent compliance $C_{r}=2 \cdot W_{r}=8,80 \cdot 10^{-3}$ Joules. Let us remind that SIMP optimization 
iterations are basically intended to minimize global compliance $\tilde{C}$. As introduced in section 3 , this global compliance is affected by the relative density field $\rho(x, y, z)$ (the symbol $\sim$ is used) and it is theoretically defined as $\tilde{C}=\{\widetilde{U}\}^{t} \cdot[\widetilde{K}] .\{\widetilde{U}\}$. Practically, it is calculated as $\tilde{C}=2 \cdot \widetilde{W}$ from the total strain energy at the last SIMP iteration $\widetilde{W}$. It is thus computed as the sum of elementary strain energies over the whole initial model as shown in Figure 11a. These elementary strain energies are affected by the SIMP relative density distribution $\rho(x, y, z)$ (symbol $\sim$ is used). In this case, $\tilde{C}=9,6 \cdot 10^{-3}$ Joules as introduced in section 3 , which obviously, is significantly higher than $C_{r}$.

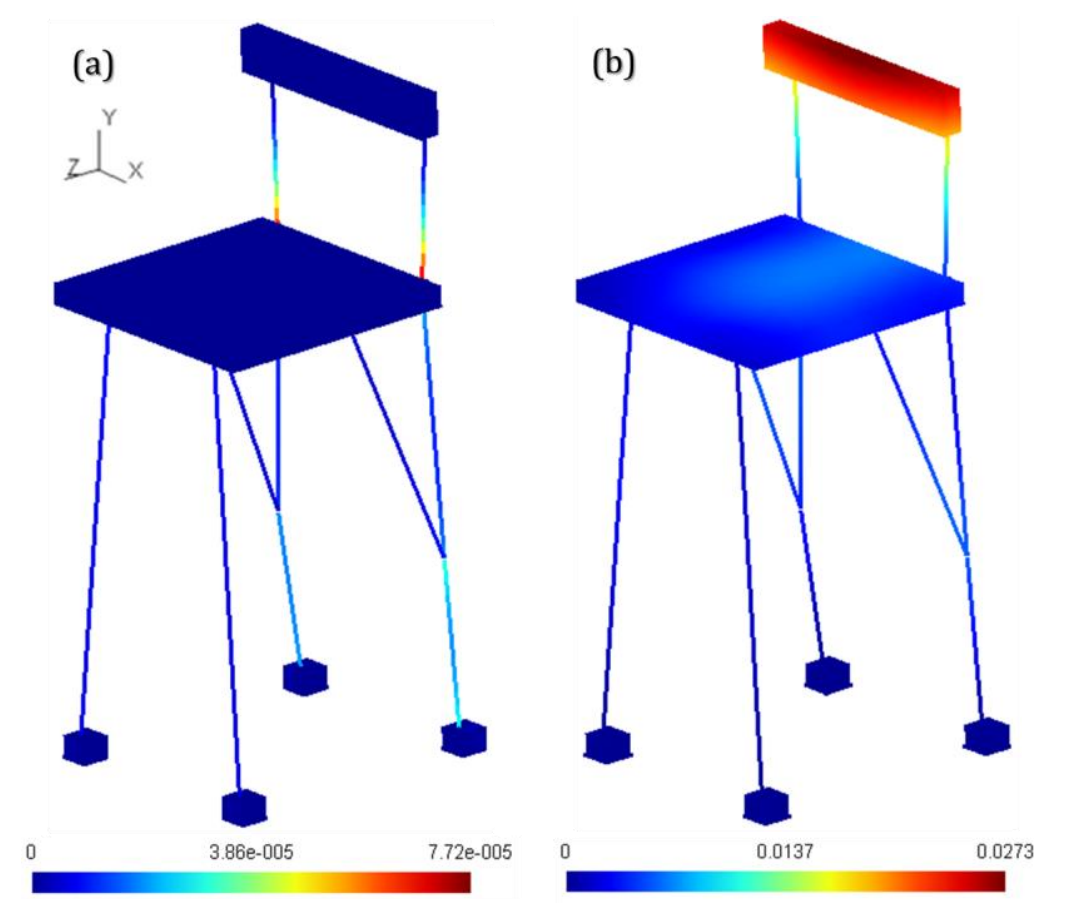

Figure 10 : Chair mixed-dimensional FEA model with the distribution of (a) elementary strain energy in Joules and (b) norm of displacement vector in $\mathrm{mm}$.

These two compliances $\tilde{C}$ and $C_{r}$ can finally be compared with a third compliance, referred to in this paper as the effective compliance $C$, as defined just below. It is important to remind here that, when comparing $\tilde{C}$ with $C$ and $C_{r}$, it is only the order of magnitude of this comparison that is meaningful since $\tilde{C}$ is affected by the relative density distribution $\rho(x, y, z)$ and by then, it does not have a physical meaning. Moreover, $\tilde{C}$ takes into account the compliance associated with elements that are not considered (for which $\rho<\rho_{t h}$ ) in the optimized model, which is not the case for $C$ and $C_{r}$. On the other hand, the comparison between $C$ and $C_{r}$ assesses how close, particularly in term of the objective function (here the compliance), the reconstructed structure is to the optimized shape provided by SIMP iterations before reconstruction.

The optimized shape is based on keeping tetrahedral elements for which $\rho \geq \rho_{\text {th }}$ and on associating to these tetrahedrons material properties of the actual material (not affected by the relative density distribution $\rho(x, y, z)$ ). The computation of the effective compliance is based on performing a new FEA simulation on the volume defined by these tetrahedrons along with non- 
design tetrahedrons. Thus, a new FEA simulation (with the same boundary conditions and loads) is performed on the volume shown in Figure 2b. In this new FEA simulation, Young's modulus $(E=69 \mathrm{GPa})$ of the actual material is considered across the whole optimized domain. This means that results of this new FEA simulation are not affected by the relative density distribution $\rho(x, y, z)$ (symbol $\sim$ is not used). Figure 11b-c show respectively the distribution of elementary strain energy and norm of the displacement vector obtained from this new FEA simulation. The effective total strain energy $W$ is computed as the sum of these elementary strain energies over the optimized model (including non-design material) and the effective compliance $C$ is defined as $C=2 \cdot W$. In this case, $C=5,46 \cdot 10^{-3}$ Joules, which can be compared with $C_{r}=8,80$. $10^{-3}$ Joules. These results show that, in this case, the reconstructed structure (Figure 7a) is more compliant than the optimized shape before reconstruction (Figure 2b), since $C_{r}$ is higher than $C$. This outcome is substantiated by the displacement (Figure 10b and Figure 11c) distribution in both optimized and reconstructed models. Indeed on these figures, it is observed for the displacement field that maximum value $\delta_{r}=0,0273 \mathrm{~mm}$ is obtained for the reconstructed model, if compared with $\delta=0,0059 \mathrm{~mm}$ for the optimized model. Figure 12 shows maximum and minimum signed $\sigma_{x}$ stress distributions across the wireframe geometry (expressed in beams local frame). These stress distributions cumulate bending and axial stresses, which are dominant stress components for all cases studied. For this example, the maximum $\sigma_{x}$ stress is $1,28 \mathrm{MPa}$.
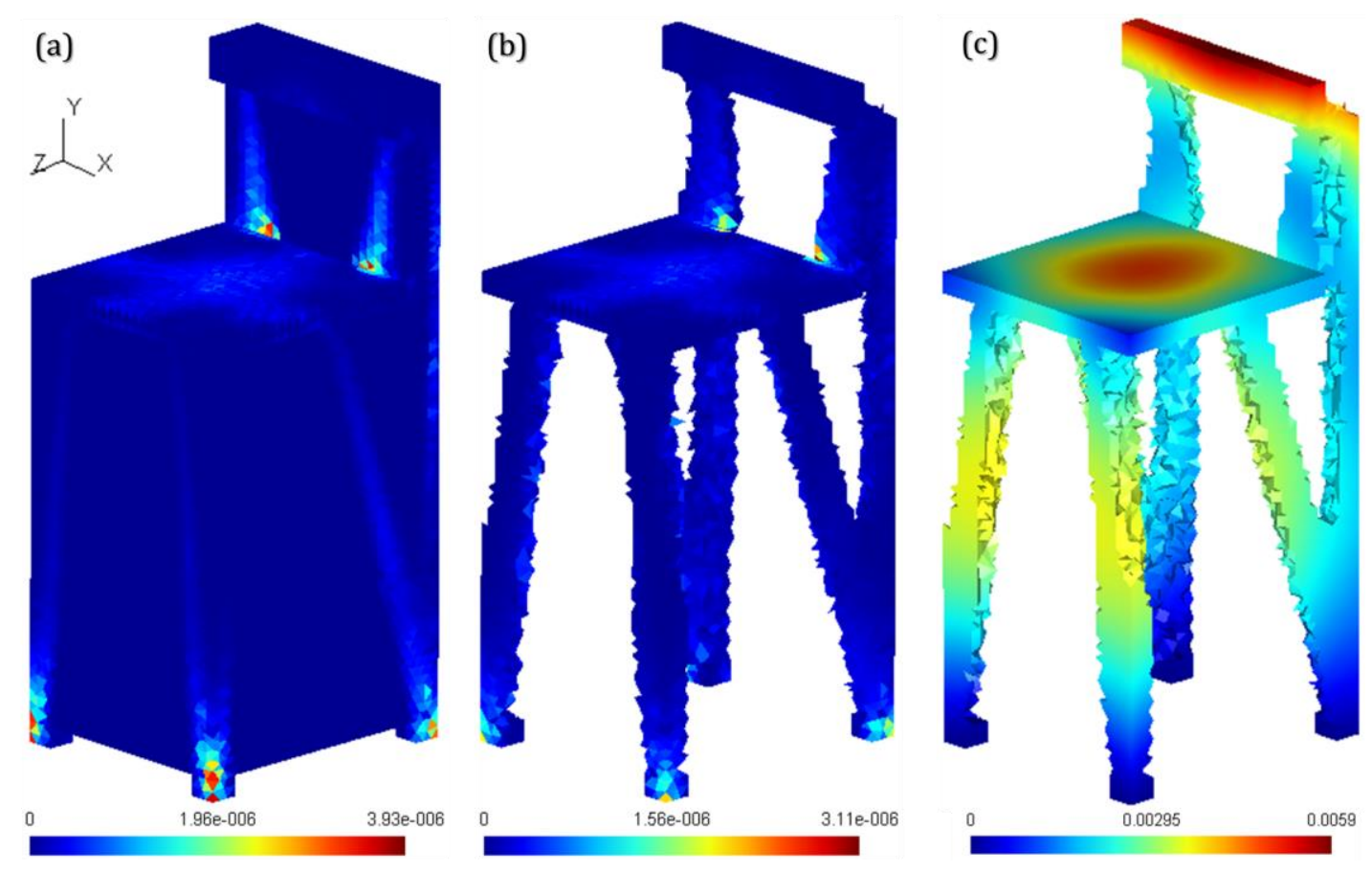

Figure 11 : Strain energy distribution in Joules in (a) the whole model after convergence of SIMP optimization, (b) the optimized model after removing void elements and (c) distribution of the norm of displacement vector in the optimized model in $\mathrm{mm}$.

Volumes obtained at different stages of the process can also be compared. It is important to remind that, unlike what is done when comparing compliances, all volumes are computed and compared without taking non-design material into account. As introduced in section 3 , this is due 
to the fact that non-design material is not affected by SIMP optimization and that, consequently, non-design volume remains constant. In this example, as introduced in section 3 , the target optimized volume is $\tilde{V}=4,72 \cdot 10^{-3} \mathrm{~m}^{3}$ and the optimized material volume obtained at the end of SIMP iterations is $\tilde{V}_{o p}=4,71 \cdot 10^{-3} \mathrm{~m}^{3}$. Volume of the optimized shape is $V_{s h}=4,84$. $10^{-3} \mathrm{~m}^{3}$ after processing non-manifold patterns as explained by [41] and before smoothing (Figure 2c) and $V_{s m}=4,83 \cdot 10^{-3} \mathrm{~m}^{3}$ after smoothing (Figure $4 \mathrm{~b}$ ). Then, volume of the reconstructed structural design once section properties are calculated (Figure $8 \mathrm{a}$ ) is $V_{r}=4,2$. $10^{-3} \mathrm{~m}^{3}$. This last result confirms that the reconstructed structure represents a good approximation of $\mathrm{TO}$ results.

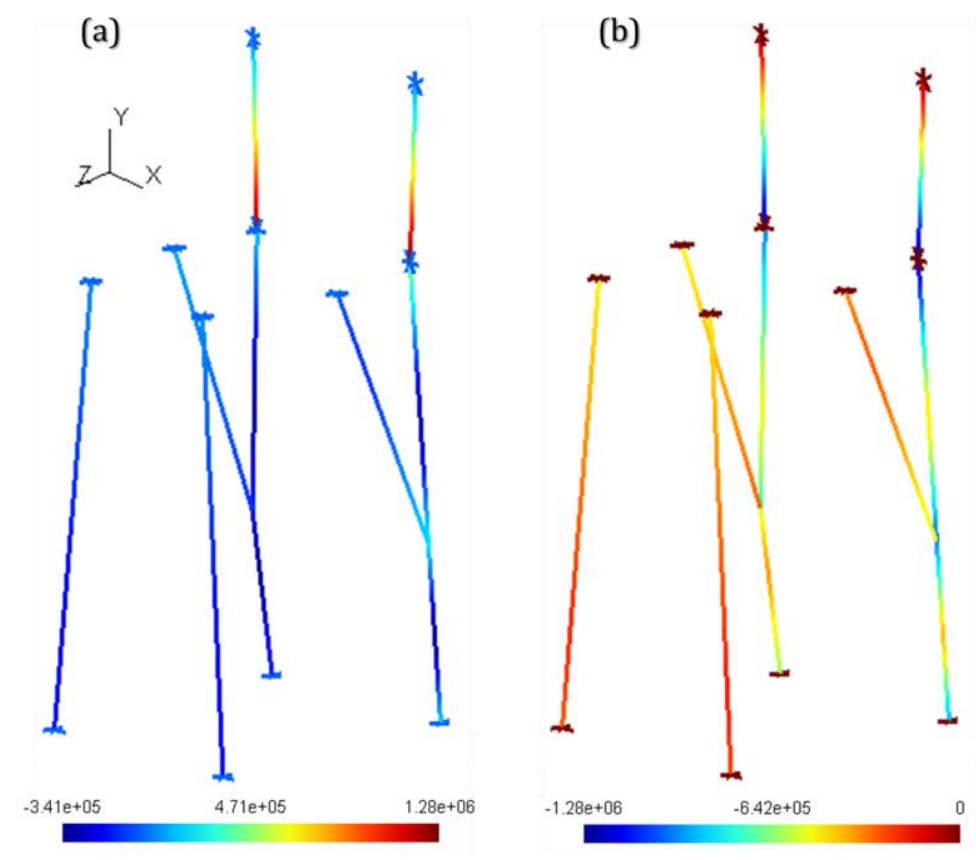

Figure 12 : Distribution of (a) maximum and (b) minimum $\sigma_{x}$ stress (in $\mathrm{N} / \mathrm{m}^{2}$ ).

\section{$5 \quad$ Results and discussions}

The effectiveness of our reconstruction strategy is demonstrated through 3 structural design examples (a cantilever beam, a footbridge and a L-bracket). In these examples, Young's modulus is $69 \mathrm{GPa}$, Poisson's ratio is 0,30. Volume fractions imposed at the beginning of SIMP optimization for these 3 cases are respectively 5\%,10\% and 4\%. These volume fractions are low, which makes that beam-like results are expected at the end of SIMP optimization. As explained in section 3, the threshold for SIMP convergence is $\Delta_{c o n v}=0,5 \%$ for all cases. As for the illustrative chair model, compliances $\tilde{C}, C$ and $C_{r}$ as well as volumes $\tilde{V}_{o p}, \tilde{V}_{s h}, V_{s m}$, and $V_{r}$ are given for each example. 


\section{$5.1 \quad \underline{\text { A Cantilever beam }}$}

The first example considered is a cantilever beam. This model has been studied by several authors in 2D [9, 13, 16-18]. The beam is shown in Figure 13a along with loads and boundary conditions applied. It is loaded with a downward pressure $\left(10 \mathrm{kN} / \mathrm{m}^{2}\right.$ in the $Y$ direction $)$ in the middle of its free end and null displacements (in $X, Y$ and $Z$ directions) are imposed at its other end. The beam model features a square section $(50 \mathrm{~mm} \times 50 \mathrm{~mm})$ and $250 \mathrm{~mm}$ length. Figure $13 \mathrm{~b}$ presents design material (in blue) and the non-design material (in red). The uniform tetrahedral mesh size used is $d_{g}=3,0 \mathrm{~mm}$ and the volume fraction is $f=5 \%$. SIMP optimization reached convergence after 36 iterations and the global compliance obtained after convergence is $\tilde{C}=3,26 \cdot 10^{-6} \mathrm{Joules}$. The optimal relative density distribution and the raw optimized result (only tetrahedrons for which relative density $\rho \geq \rho_{t h}=0,35$ are considered) are respectively shown in Figure 14a and Figure 14b.
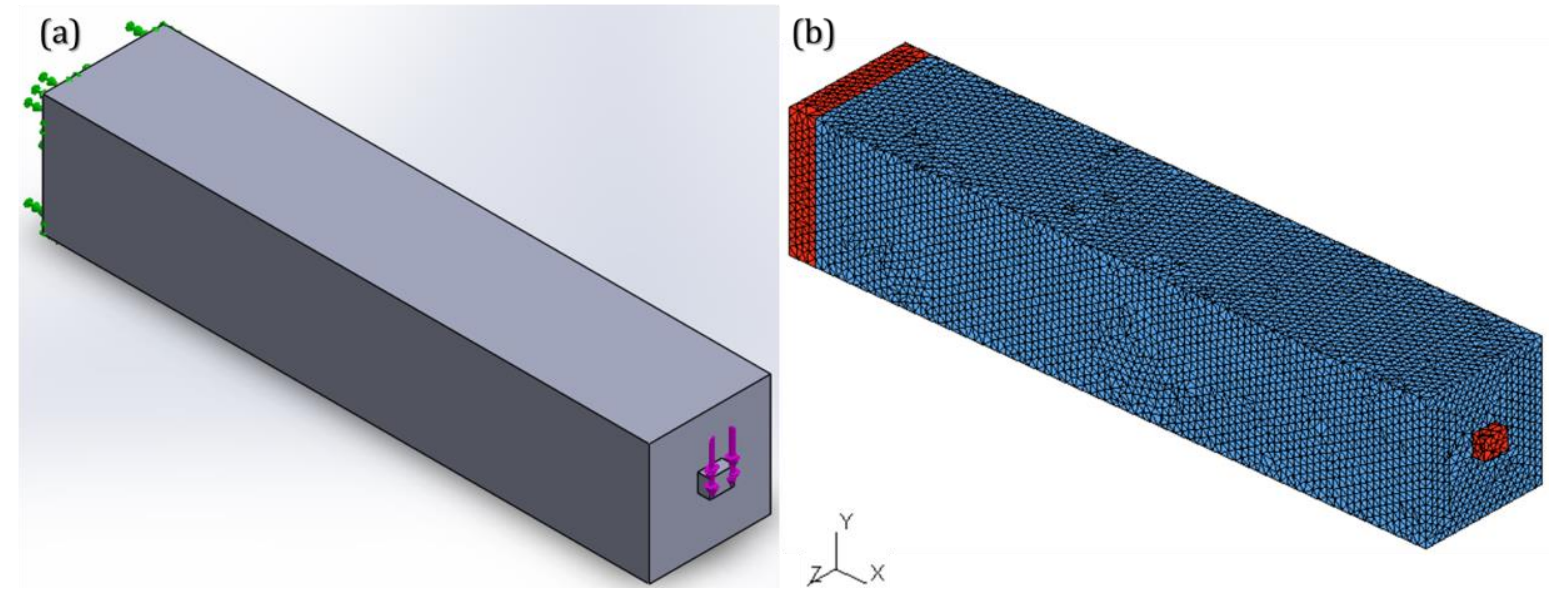

Figure 13 : Cantilever (a) geometry, loads and boundary conditions (b) design (in blue) and nondesign (in red) material using a uniform mesh size $\left(d_{g}=3,0 \mathrm{~mm}\right)$.
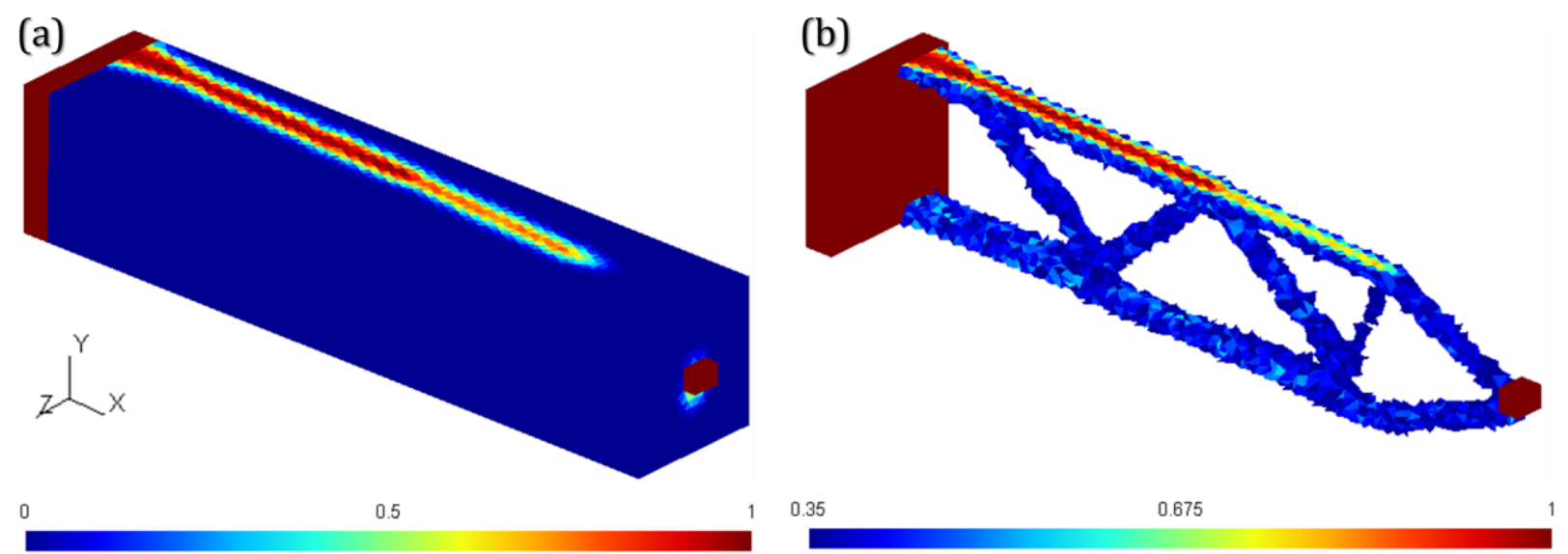

Figure 14 : Cantilever (a) relative density distribution after convergence of SIMP optimization (b) raw optimized shape. 
The volume at the end of the SIMP process and the volume of the rough optimal design after processing non-manifold patterns, are respectively $\tilde{V}_{o p}=2,9 \cdot 10^{-5} \mathrm{~m}^{3}$ and $\tilde{V}_{s h}=3,0 \cdot 10^{-5} \mathrm{~m}^{3}$. The optimal shape is illustrated in Figure 15a. The volume of the smoothed shape (non-design material excluded) is $V_{s m}=2,7 \cdot 10^{-5} \mathrm{~m}^{3}$, and the associated reconstructed volume is $V_{r}=2,4$. $10^{-5} \mathrm{~m}^{3}$. Figure $15 \mathrm{~b}-\mathrm{c}$ illustrate the skeleton structure before and after normalization along with non-design material schematized as solid geometry in yellow.

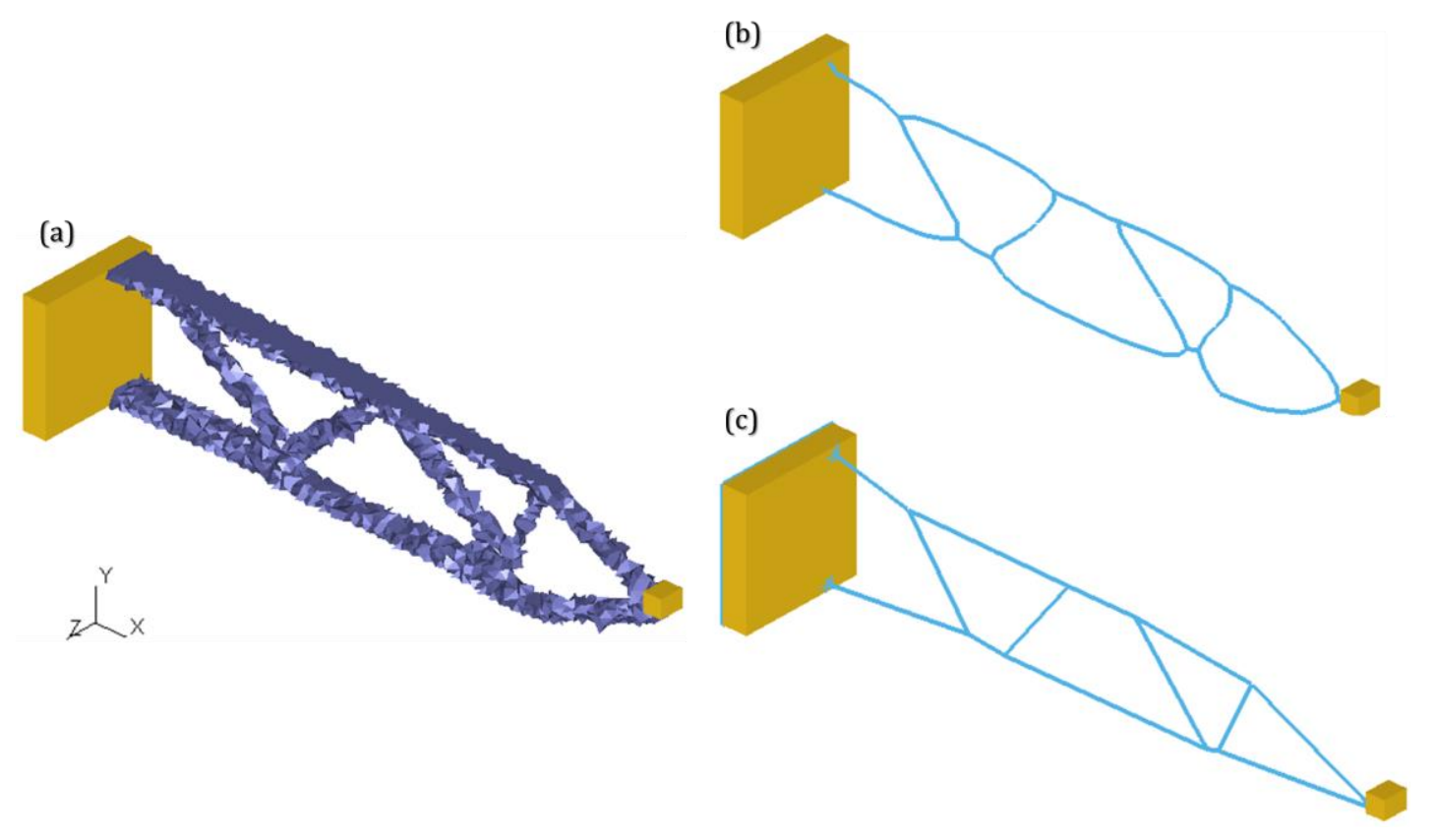

Figure 15 : Cantilever (a) rough optimized shape (b) skeleton before normalization (c) skeleton after normalization and connection with mini-beams.

It can be seen from Figure 15 that instead of 11 beams, as expected from the rough TO result, the curve skeleton features 14 beams. As a matter of fact, 3 beams have been reconstructed in place of junction points in the skeleton. This configuration illustrates a potential improvement of our strategy. Indeed, as suggested in [15], rules, such as closest nodes merging and discarding specific nodes or beams, could be introduced in our approach to generate more meaningful curve skeletons.

Figure 16a-b show results obtained (norm of displacement vector in $\mathrm{mm}$ and elementary strain energy in Joules) from the mixed-dimensional FEA model. Compliances of the reconstructed beam-solid structure and after 3D FEA applied to the rough optimized model are respectively $C_{r}=2,40 \cdot 10^{-6}$ Joules and $C=9,14 \cdot 10^{-7}$ Joules. It is worth noting that these values are quite close, despite the fact that, as introduced just above, topologies of the rough optimized model (Figure 15a) and of the reconstructed structure (Figure 15c) are not exactly the same. 

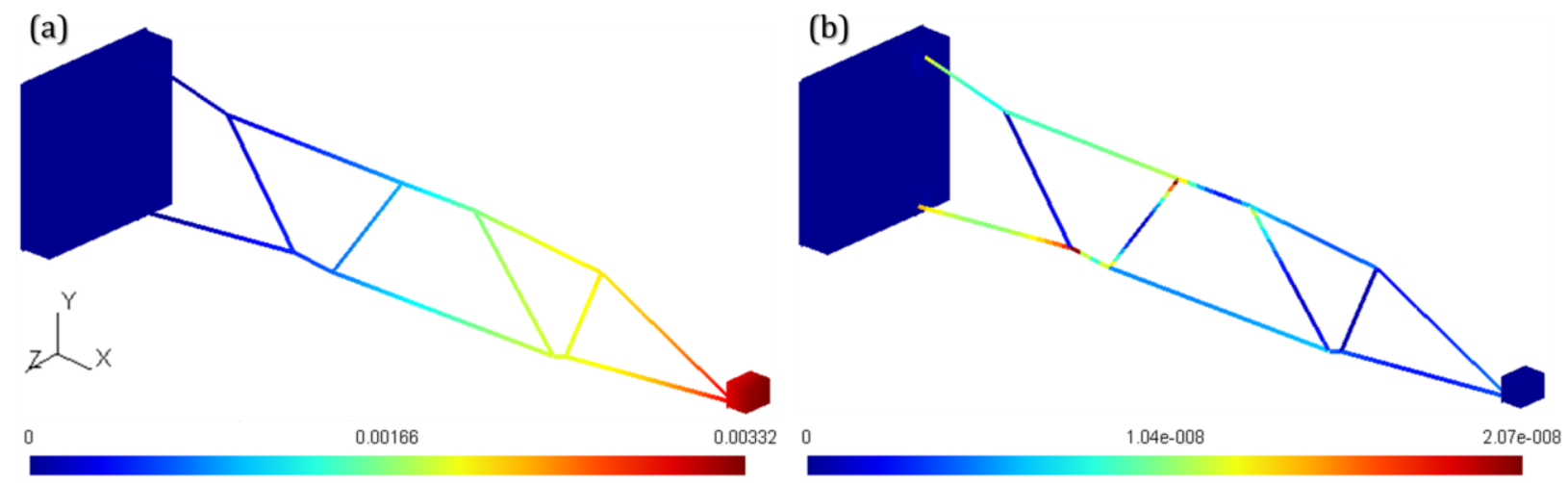

Figure 16 : Cantilever mixed-dimensional FEA model with the distribution of (a) norm of displacement vector in mm (b) elementary strain energy in Joules.

\subsection{A Footbridge}

The second example features a footbridge. The initial dimensions are $80 \mathrm{~mm} \times 90 \mathrm{~mm} \times$ $400 \mathrm{~mm}$. As shown in Figure 17a, it is loaded with a downward pressure $\left(10 \mathrm{kN} / \mathrm{m}^{2}\right.$ in the $Y$ direction) and null displacements (in $X, Y$ and $Z$ directions) are imposed at the anchors. Nondesign (in red) and design material (in blue) are presented in Figure 17b, using a uniform size for the tetrahedral mesh used for TO $\left(d_{g}=4,5 \mathrm{~mm}\right)$. In this case, we set $f=10 \%$ as volume fraction. Figure 18a-b respectively present the optimal relative density distribution after TO, and the raw optimized shape where only elements for which relative density $\rho \geq \rho_{t h}=0,40$ are considered.
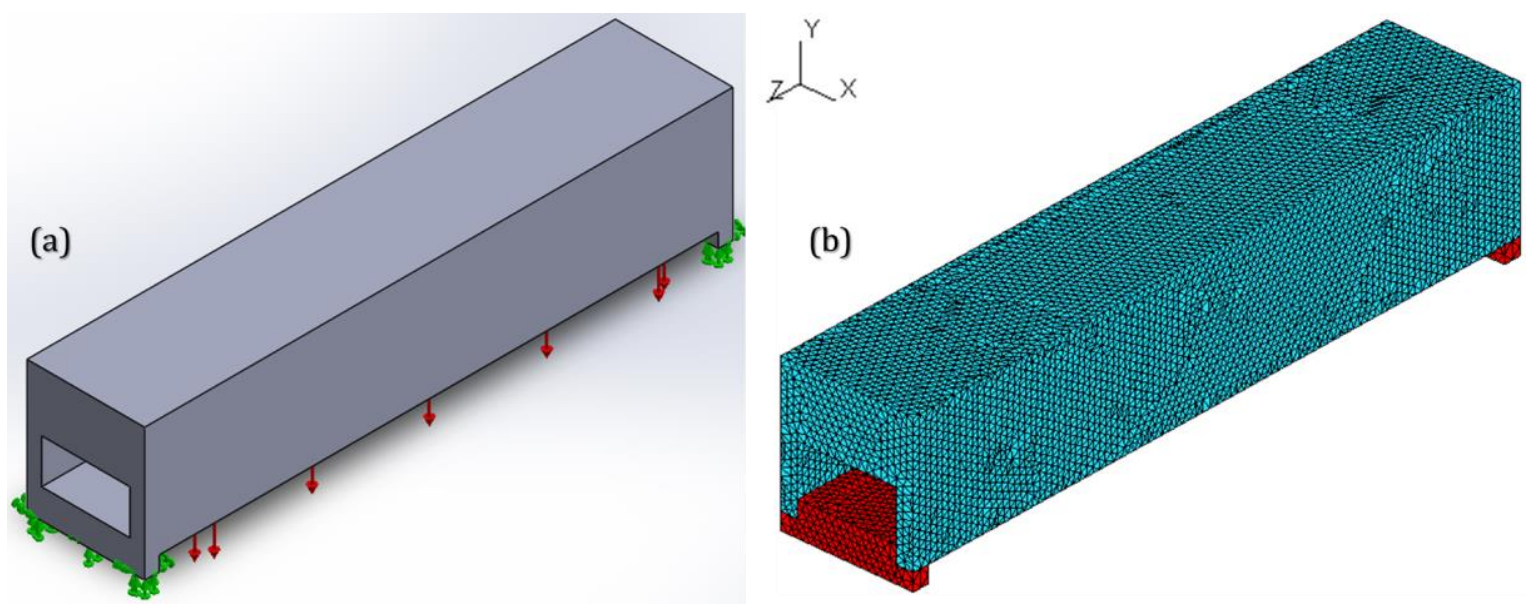

Figure 17 : Footbridge (a) geometry, loads and boundary conditions (b) design (in blue) and nondesign (in red) material using a uniform mesh size $\left(d_{g}=4,5 \mathrm{~mm}\right)$. 

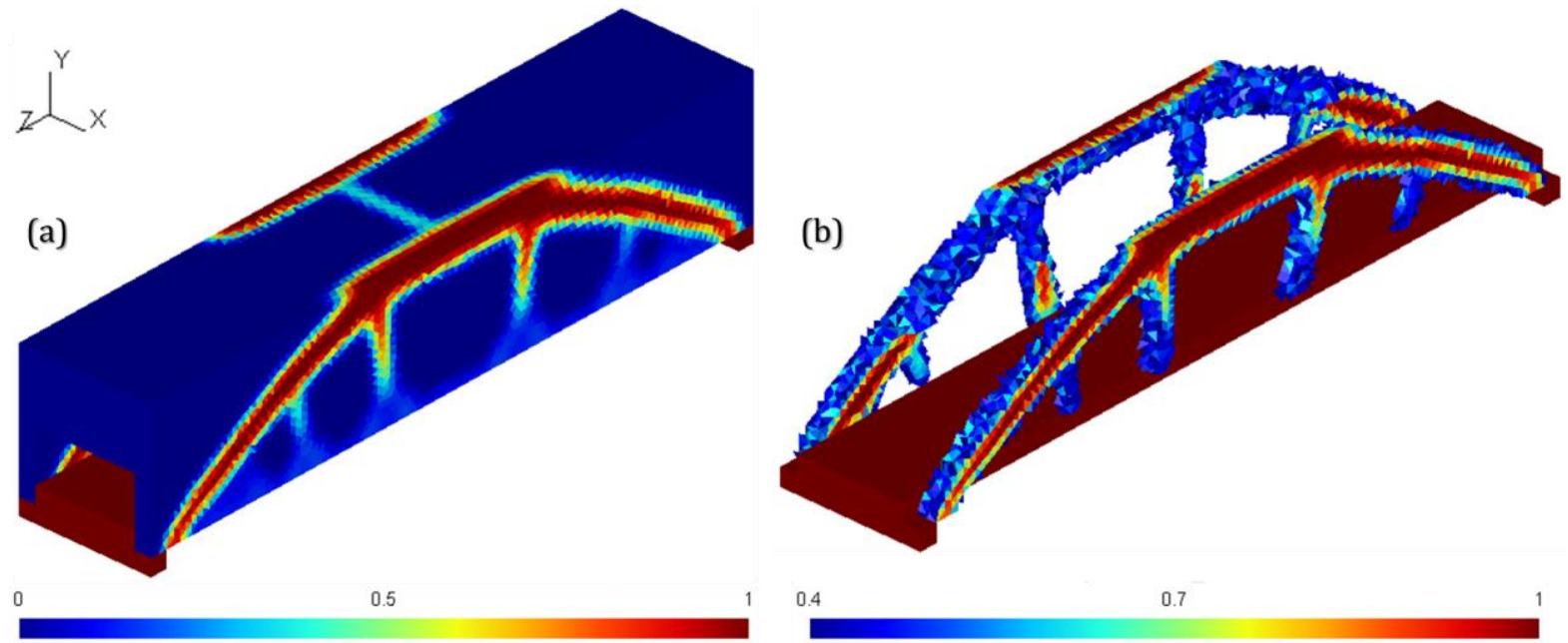

Figure 18 : Footbridge (a) the relative density distribution after convergence of SIMP optimization (b) raw optimized shape.

Figure 19a shows the rough shape of the optimized result (in grey) and the non-design domain (in yellow). Volumes at the end of the optimization and after the rough shape extraction are respectively $\tilde{V}_{o p}=1,6 \cdot 10^{-4} \mathrm{~m}^{3}$ and $\tilde{V}_{s h}=1,6 \cdot 10^{-4} \mathrm{~m}^{3}$. In addition, the volume after smoothing (also with non-design material excluded) and the associated reconstructed volume are respectively $V_{s m}=1,5 \cdot 10^{-4} \mathrm{~m}^{3}$ and $V_{r}=1,4 \cdot 10^{-4} \mathrm{~m}^{3}$. SIMP optimization converged after 24 iterations and the global compliance obtained is $\tilde{C}=1,68 \cdot 10^{-3}$ Joules.

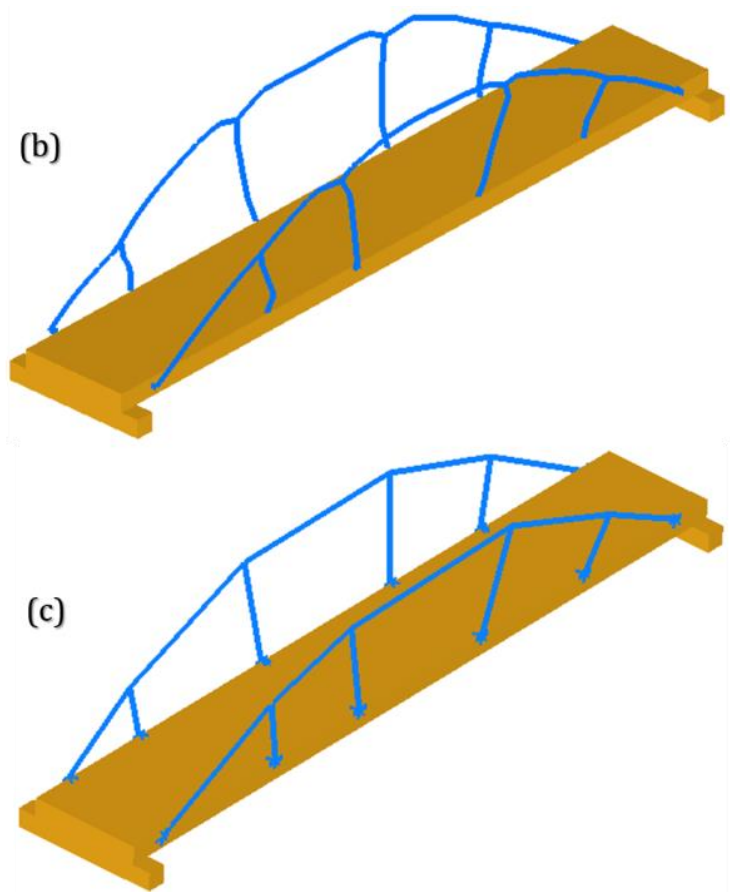

Figure 19 : Footbridge (a) rough optimized shape (b) skeleton before normalization (c) skeleton after normalization and connection with mini-beams. 
Figure 20 shows results obtained (norm of displacement vector in $\mathrm{mm}$ and elementary strain energy in Joules) from the mixed-dimensional FEA model. Compliances of the reconstructed beam-solid structure and after the 3D FEA applied to the rough optimized model are respectively $C_{r}=7,45 \cdot 10^{-4}$ Joules and $C=5,97 \cdot 10^{-4}$ Joules. In this case, reconstructed compliance $C_{r}$ is about half of effective compliance $C$.
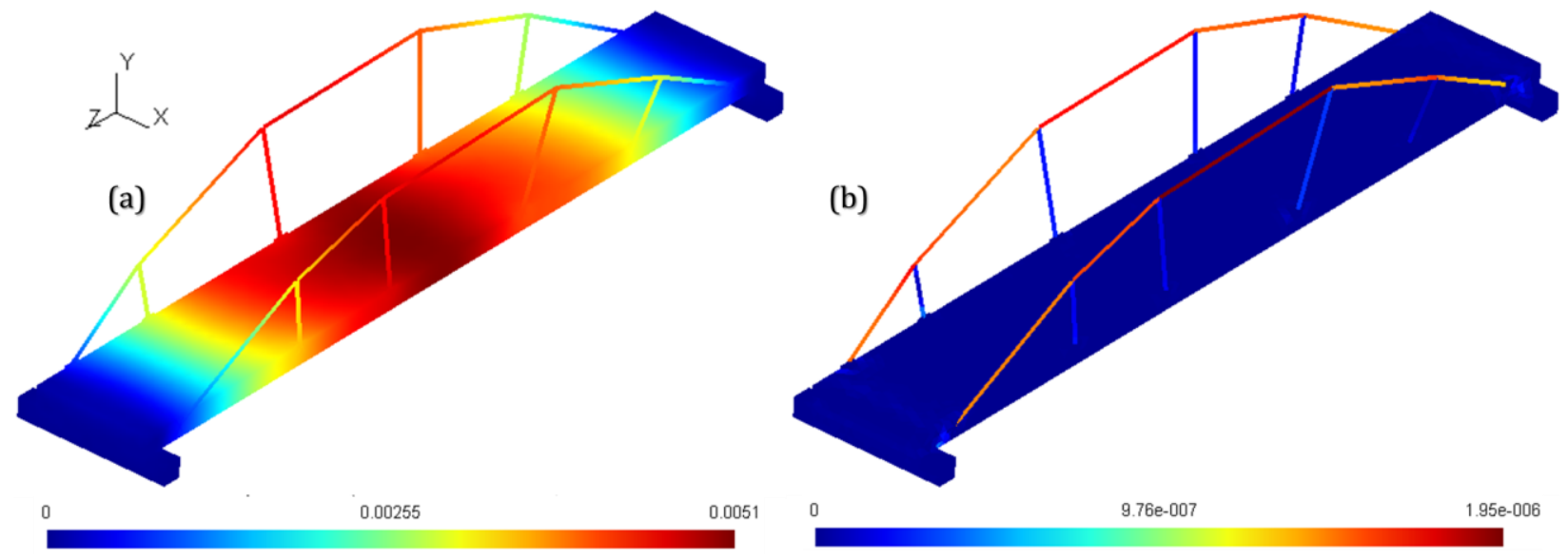

Figure 20 : Footbridge mixed-dimensional FEA model with the distribution of (a) norm of displacement vector in $\mathrm{mm}$ (b) elementary strain energy in Joules.

\section{$5.3 \underline{\text { A L-bracket }}$}

The third example considered is a L-bracket (see Figure 21).
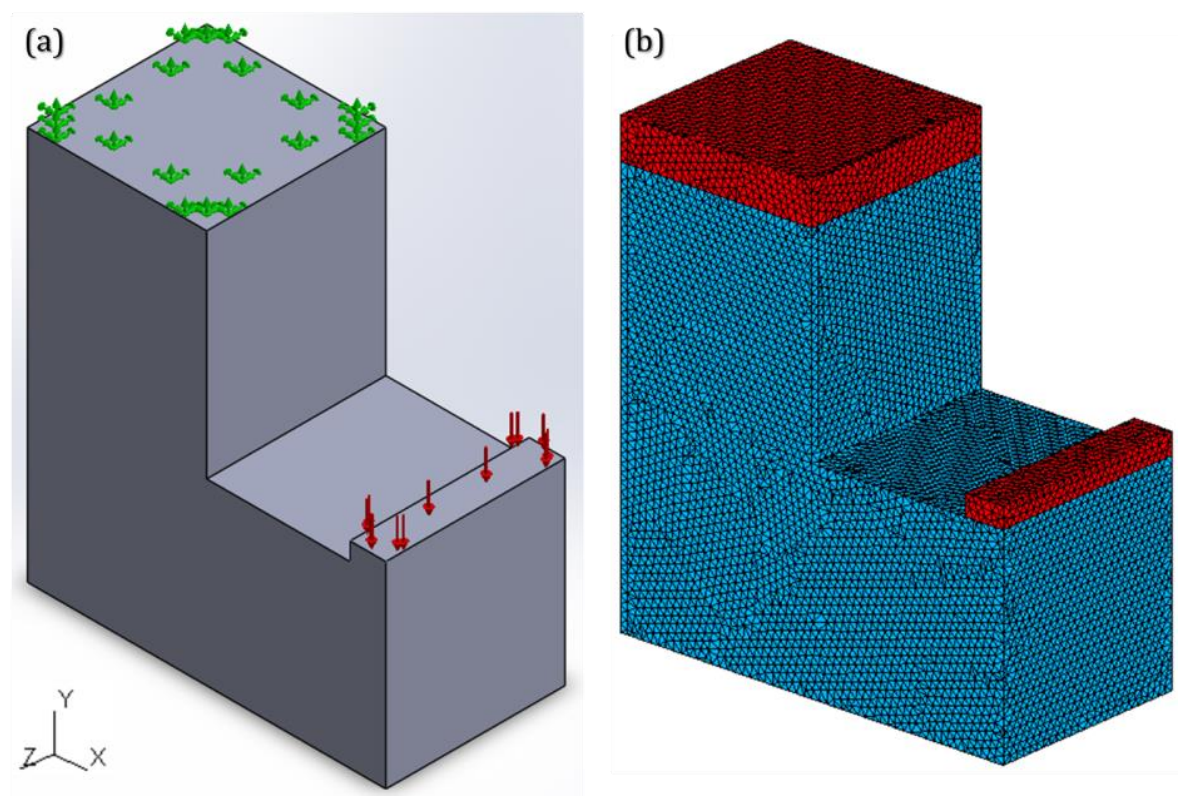

Figure 21 : L-bracket (a) geometry, loads and boundary conditions (b) design (in blue) and nondesign (in red) material using a uniform mesh size $\left(d_{g}=40 \mathrm{~mm}\right)$. 
The initial dimensions are $2 m \times 2 m \times 1 \mathrm{~m}$. It is loaded with a downward pressure $\left(10^{6} \mathrm{~N} / \mathrm{m}^{2}\right)$ in the $Y$ direction and null displacements (in $X, Y$ and $Z$ directions) are imposed at its upper left side (see Figure 21a). As shown in Figure 21b, non-design material (in red) is distributed around anchored and loaded surfaces. Tetrahedrons with a uniform mesh size $\left(d_{g}=\right.$ $40 \mathrm{~mm}$ ) are used for SIMP optimization. As introduced above, $f=4 \%$ is used as volume fraction. SIMP optimization reached convergence after 28 iterations and compliance of the whole model obtained is $\tilde{C}=1100$ Joules. The optimal relative density distribution at the end of SIMP iterations and the raw optimized result (only tetrahedrons for which relative density $\rho \geq$ $\rho_{t h}=0,40$ are considered) are respectively shown in Figure 22a-b.
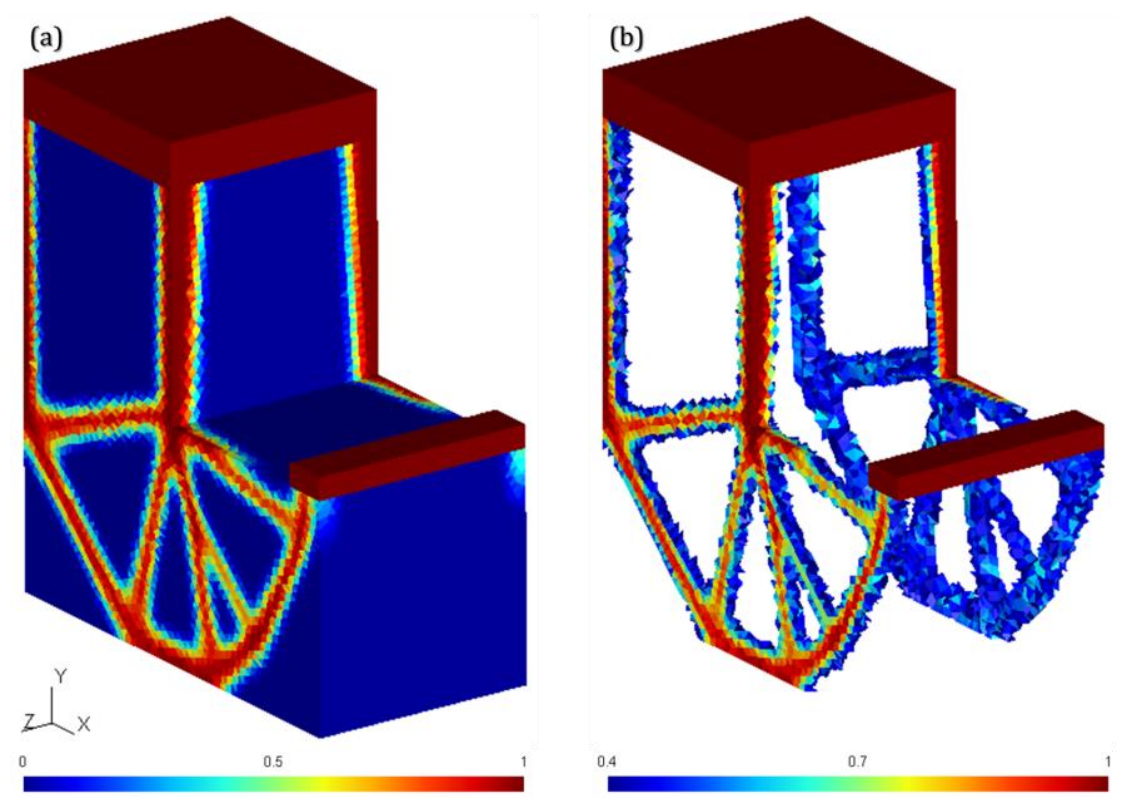

Figure 22 : L-bracket (a) relative density distribution after convergence of SIMP optimization (b) raw optimized shape.

Volume at the end of SIMP optimization is $\tilde{V}_{o p}=1,19 \cdot 10^{-1} \mathrm{~m}^{3}$. After discarding elements for which relative density is lower than $\rho_{t h}$, volume of the rough optimal design (in grey in Figure 23a) is $V_{s h}=1,25 \cdot 10^{-1} \mathrm{~m}^{3}$. Volume of the smoothed optimal shape (non-design material excluded) is $V_{s m}=1,09 \cdot 10^{-1} \mathrm{~m}^{3}$, and the associated reconstructed volume is $V_{r}=1,18$. $10^{-1} \mathrm{~m}^{3}$. Figure 23b-c respectively illustrate skeletons before and after normalization along with non-design material as solid geometry in yellow.

Figure 24 shows results obtained (norm of displacement vector in $\mathrm{mm}$ and elementary strain energy in Joules) from the mixed-dimensional FEA model (see Figure 23c) when applying the same boundary conditions and loads as in Figure 21a. This figure reveals that for the displacement field, a maximum value $\delta_{b r}=5,6 \mathrm{~mm}$ is obtained. Compliance of the reconstructed beam-solid structure is $C_{r}=2 \cdot W_{r}=753 \mathrm{Joules}$, and compliance obtained after performing 3D FEA on the rough optimized model (with the same boundary conditions and loads applied) is $C=528$ Joules. The same trend as for previous examples can be seen, the 
reconstructed structure is more flexible than the optimized structure. Main results of this last case are also summarized in Table 1.
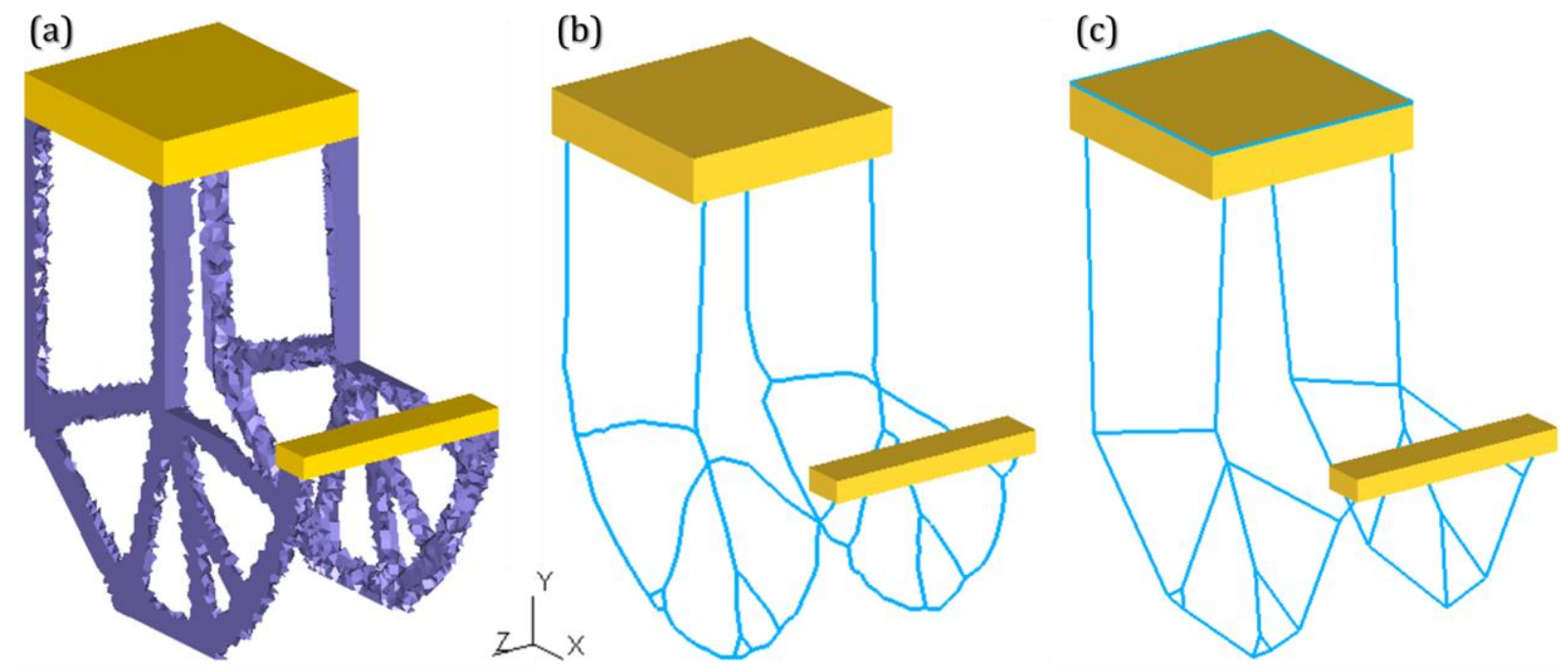

Figure 23 : L-bracket (a) rough optimized shape (b) skeleton before normalization (c) skeleton after normalization and connection with mini-beams.
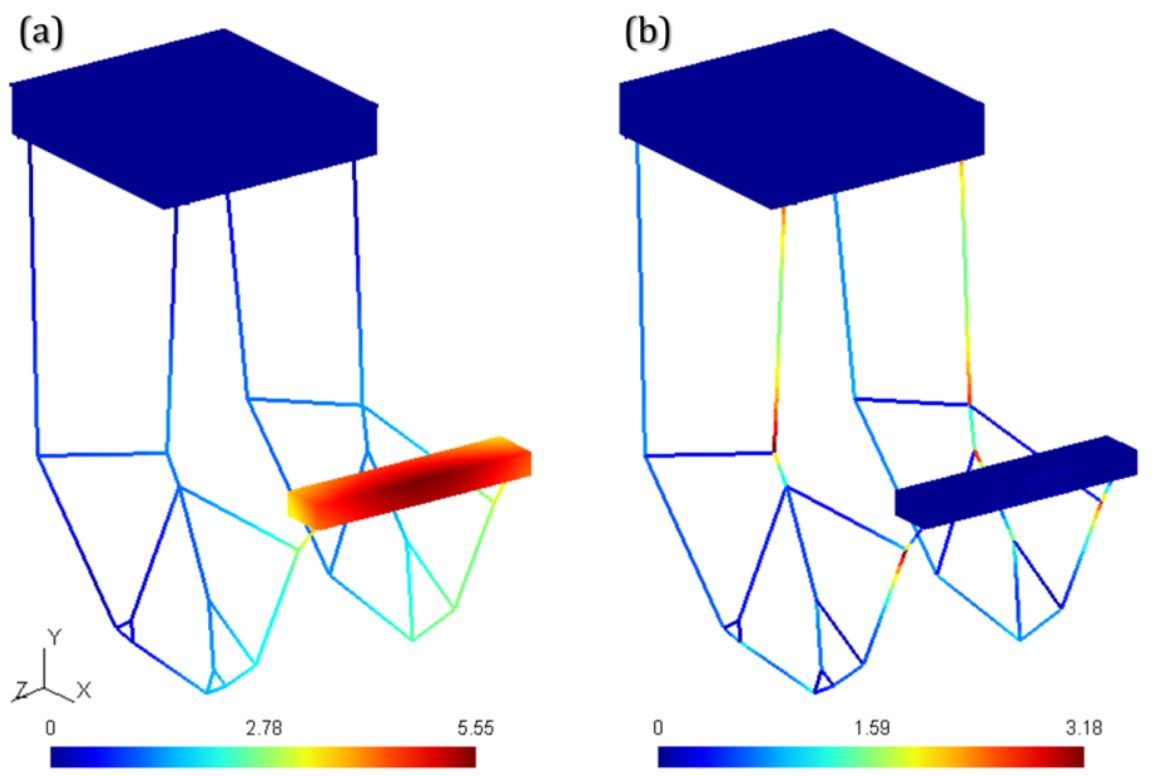

Figure 24 : L-bracket mixed-dimensional FEA model with the distribution of (a) norm of displacement vector in $\mathrm{mm}$ (b) elementary strain energy in Joules.

\subsection{Summary of results}

Table 1 provides data for quantitative validation of all examples proposed in this section. This table include skeleton characteristics, volumes, and compliances for each example used to illustrate our approach. 


\begin{tabular}{|c|c|c|c|c|}
\hline & Chair & Cantilever & Footbridge & L-Bracket \\
\hline \multicolumn{5}{|c|}{ Some characteristics of the beam-solid model } \\
\hline Number of junction points & 2 & 3 & 8 & 20 \\
\hline Number of branches (straight beams) & 10 & 14 & 18 & 34 \\
\hline Number of beam-solid connections & 12 & 3 & 12 & 6 \\
\hline Total number of mini-beams & 70 & 17 & 74 & 36 \\
\hline \multicolumn{5}{|c|}{ Volume of design material (non-design material excluded) in $m^{3}$} \\
\hline Extraction threshold $: \rho_{t h}$ & 0,45 & 0,35 & 0,40 & 0,40 \\
\hline Volume of the initial design material $: V_{d}$ & $5,90 \cdot 10^{-2}$ & $6,00 \cdot 10^{-4}$ & $1,60 \cdot 10^{-3}$ & 3,00 \\
\hline Volume objective of the optimization $: \tilde{V}$ & $4,72 \cdot 10^{-3}$ & $3,00 \cdot 10^{-5}$ & $1,60 \cdot 10^{-4}$ & $1,20 \cdot 10^{-1}$ \\
\hline Volume at the end of the optimization: $V_{o p}$ & $4,71 \cdot 10^{-3}$ & $2,98 \cdot 10^{-5}$ & $1,60 \cdot 10^{-4}$ & $1,19 \cdot 10^{-1}$ \\
\hline Volume after shape extraction at $\rho_{t h}: V_{s h}$ & $4,84 \cdot 10^{-3}$ & $3,07 \cdot 10^{-5}$ & $1,60 \cdot 10^{-4}$ & $1,25 \cdot 10^{-1}$ \\
\hline Volume after smoothing $: V_{s m}$ & $4,83 \cdot 10^{-3}$ & $2,74 \cdot 10^{-5}$ & $1,50 \cdot 10^{-4}$ & $1,09 \cdot 10^{-1}$ \\
\hline Volume after reconstruction $: V_{r}$ & $4,21 \cdot 10^{-3}$ & $2,40 \cdot 10^{-5}$ & $1,40 \cdot 10^{-4}$ & $1,18 \cdot 10^{-1}$ \\
\hline Ratio $V_{s h} / V_{r}$ & 1,15 & 1,28 & 1,14 & 1,06 \\
\hline Volume fraction prescription & $8,0 \%$ & $5,0 \%$ & $10,0 \%$ & $4,0 \%$ \\
\hline Volume fraction after smoothing & $8,2 \%$ & $4,6 \%$ & $9,4 \%$ & $3,6 \%$ \\
\hline Volume fraction after reconstruction & $7,1 \%$ & $4,0 \%$ & $8,8 \%$ & $3,9 \%$ \\
\hline \multicolumn{5}{|c|}{ Compliance of the structure (non-design material included) in Joules } \\
\hline Global compliance : $\tilde{C}$ & $9,60 \cdot 10^{-3}$ & $3,26 \cdot 10^{-6}$ & $1,68 \cdot 10^{-3}$ & 1100 \\
\hline Effective compliance : $C$ & $5,46 \cdot 10^{-3}$ & $9,14 \cdot 10^{-7}$ & $5,97 \cdot 10^{-4}$ & 528 \\
\hline Reconstructed compliance : $C_{r}$ & $8,80 \cdot 10^{-3}$ & $2,40 \cdot 10^{-6}$ & $7,45 \cdot 10^{-4}$ & 753 \\
\hline Ratio $C / C_{r}$ & 0,62 & 0,40 & 0,80 & 0,70 \\
\hline
\end{tabular}

Table 1 : Summary of results.

In the 4 cases presented, the rough optimized shape is well interpreted into a beam-like CAD solid design using our strategy. A look at values obtained for the ratio $V_{s h} / V_{r}$ for each case, shows that reconstructed volumes are consistent with rough optimized design volumes. It can also be observed that there is a difference between $C_{r}$ and $C$. Indeed, since $C<C_{r}$, reconstructed beam structures tend to be less stiff than rough optimized $3 \mathrm{D}$ results, which is not surprising. Indeed, the normalization approximates each branch of the optimized model as a straight beam with a constant mean circular section along its length when in fact, the optimized shape is, in general, neither straight nor circular, nor with constant section along its length. This adds to the fact that the real cross section shape of the beam can be stiffer than the simplistic circular shape, and that approximating a curve as a straight line results in a loss of length, which means in a loss of volume. Moreover, when connecting normalized skeletons with non-design material, minibeams are introduced only at a connection node, while it should be at the whole interface between the optimized and the non-design domains. This influences rigidity of the reconstructed structure. Furthermore, cross section calculation for each beam of curve skeletons (before normalization) considers skeletons as centered, which is not the case at junction and end points [42]. In fact, curve skeletons are generally not ideally generated at junction and end points. This affects cross-section computation and, by the way, compliance in each beam. Besides, one should not expect reconstructed and rough optimal structures to behave identically since one is 
made with straight uniform beams and the other with general 3D solid shapes that do not withstand stresses exactly in the same way.

\section{Stress-based design after SIMP optimization}

Considering the last example (L-Bracket) we introduce, in this section, a new strategy, based on adjusting SIMP volume fraction to fulfill maximum stress objectives (see Figure 26 below).

Figure 25 shows distributions of $\sigma_{x}$ maximum and minimum stress (cumulative of axial and bending stress components) along with that of $\tau_{\mathrm{xy}}$ and $\tau_{\mathrm{xz}}$ shear stress.
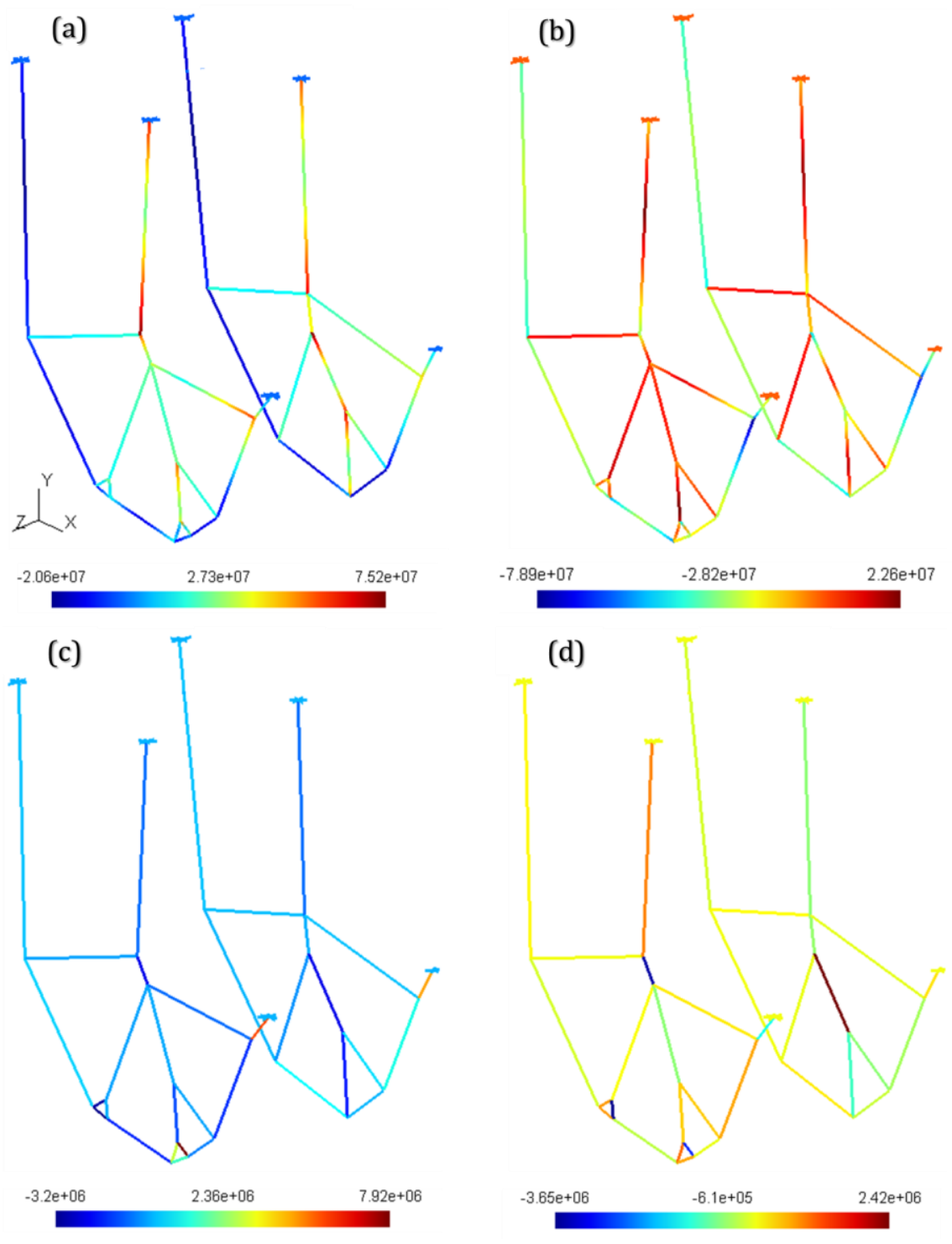

Figure 25 : L-bracket with $f=4 \%$. Distribution of (a) maximum $\sigma_{x}$ stress (b) minimum $\sigma_{x}$ stress (c) and (d) $\tau_{x y}$ and $\tau_{x z}$ shear stress (in $N / m^{2}$ ).

Since these stress components are computed for beam elements, all stress components are expressed in beams local frames. For the L-Bracket with $4 \%$ volume fraction, maximum $\sigma_{x}$ 
stress is $79 M P a$ while maximum shear stress is $7,9 M P a$. Since, in this case, shear stress can be neglected with respect to axial and bending stress, von Mises stress $\sigma_{V M}$ is assimilated with $\sigma_{x}$ stress. Thus $\sigma_{V M} \approx\left|\sigma_{x}\right|$. Thus, maximum von Mises stress is considered as $\sigma_{V M}^{\max } \approx 79 \mathrm{MPa}$ since maximum $\sigma_{x}$ stress is $75 \mathrm{MPa}$ (see Figure 25a) and minimum $\sigma_{x}$ stress is $-79 \mathrm{MPa}$ (see Figure 25b) which makes that maximum $\left|\sigma_{x}\right|$ is $79 M P a$. Considering that yield stress is $S_{y}=$ $135 \mathrm{MPa}$ (aluminum) and that targeted safety factor is 2 , the allowable stress is $\sigma_{a}=S_{y} / S F=$ $0,5 \cdot 135=67,5 \mathrm{MPa}$. Thus, for this design, $\max$ von Mises stress obtained $(79 \mathrm{MPa})$ is too high since it exceeds allowable stress $\sigma_{a}=67,5 \mathrm{MPa}$.

From this result, two options can be considered to fulfill stress objective. The first option is using a classical beam structure optimization process (like the one presented in [5]) based on keeping the same structure topology and applying an optimization based on adjusting beam cross section properties (in this case radii of normalized beam cross sections) to fulfill stress objectives.

In this paper, we introduce a second option, which consists in automatically adjusting SIMP volume fraction and re-applying the whole process to fulfill these stress objectives. This new iterative process is presented in Figure 26. In the case of the L-Bracket example, SIMP volume fraction is increased from $f=4 \%$ to $f_{2}=5 \%$.

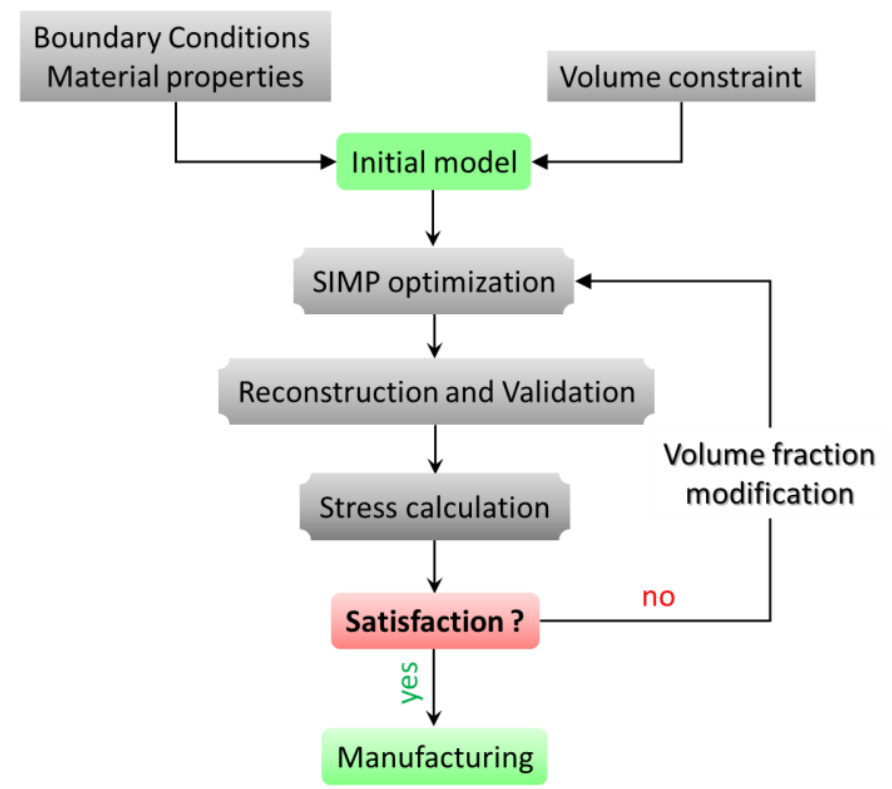

Figure 26 : Loop on the volume fraction based on stress results.

For $f_{2}=5 \%$, SIMP optimization converges after 27 iterations and compliance of the whole model obtained is $\tilde{C}_{2}=784$ Joules (compared to $\tilde{C}=1100$ Joules for $f=4 \%$ ). The optimal relative density distribution at the end of SIMP iterations and the raw optimized result (only tetrahedrons for which relative density $\rho \geq \rho_{t h}=\rho_{t h 2}=0,40$ were considered) are respectively shown in Figure 27a-b. Volume at the end of the SIMP optimization, volume of the rough optimal design, volume of the smoothed optimal shape (non-design material excluded), and volume of the reconstructed beam geometry are respectively $\tilde{V}_{o p 2}=1,49 \cdot 10^{-1} \mathrm{~m}^{3}, V_{s h 2}=$ $1,59 \cdot 10^{-1} \mathrm{~m}^{3}, \quad V_{s m 2}=1,48 \cdot 10^{-1} \mathrm{~m}^{3}, \quad$ and $V_{r 2}=1,52 \cdot 10^{-1} \mathrm{~m}^{3}$. Compliance of the reconstructed beam-solid structure is $C_{r 2}=2 \cdot W_{r 2}=569$ Joules (compared with $C_{r}=$ 
753 Joules for $f=4 \%$ ), and the compliance obtained after performing 3D FEA on the rough optimized model is $C_{2}=405$ Joules (compared with $C=528$ Joules for $f=4 \%$ ).
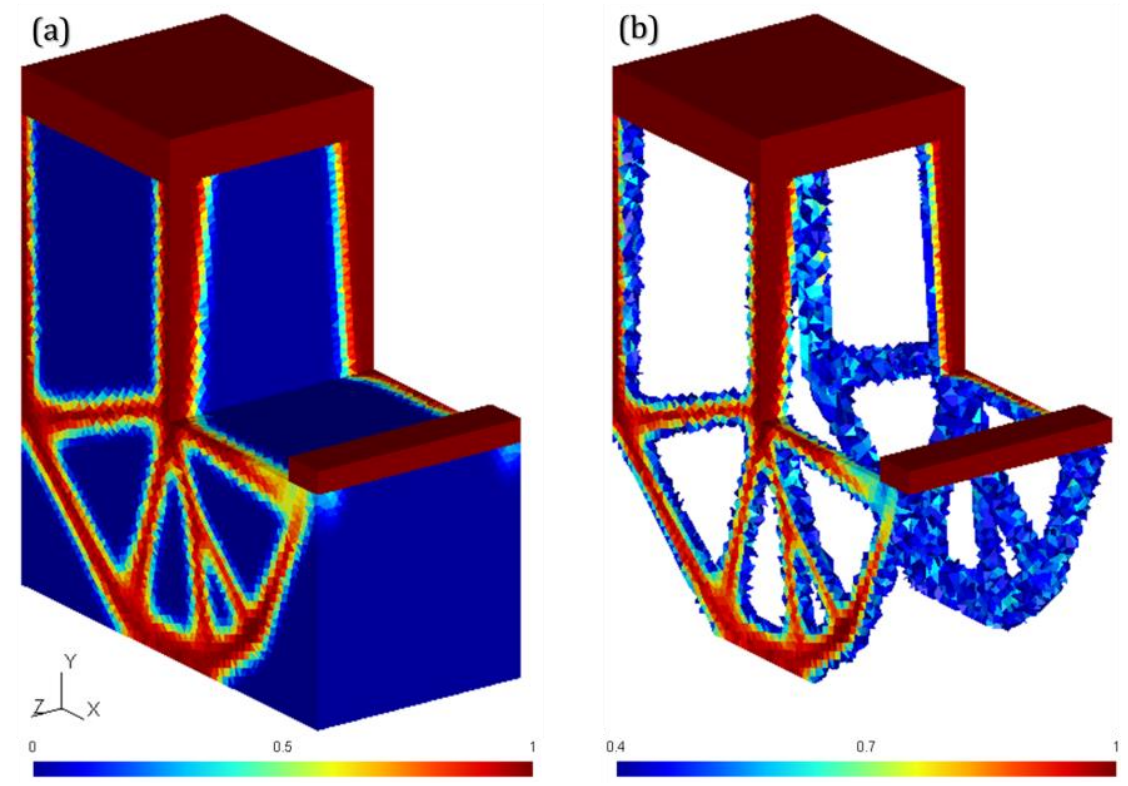

Figure 27 : L-bracket problem with $f_{2}=5 \%$. (a) Relative density distribution after convergence of SIMP optimization (b) raw optimized shape.

For comparison with results with $f=4 \%$, Figure 28 shows distributions of displacement and elementary strain energy. This figure shows that the displacement field reaches a maximum value $\delta_{b r 2}=4,1 \mathrm{~mm}$, which can be compared with $\delta_{b r}=5,6 \mathrm{~mm}$ for $f=4 \%$.
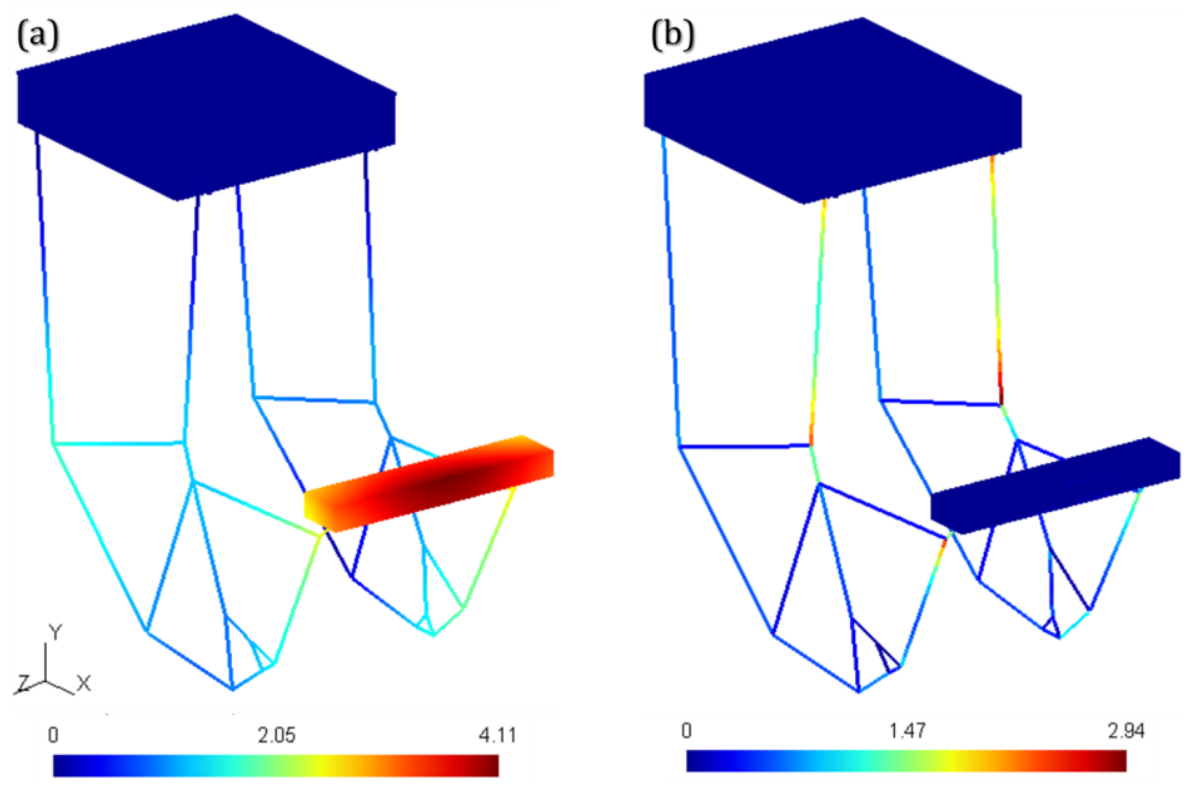

Figure 28 : L-bracket problem with $f_{2}=5 \%$. Mixed-dimensional FEA model with the distribution of (a) norm of displacement vector in $m m$ (b) elementary strain energy in Joules. 
Figure 29 shows maximum and minimum $\sigma_{x}$ stress distributions, which shows that maximum von Mises stress is $\sigma_{V M 2}^{\max } \approx 66 \mathrm{MPa}$ (compared with $\sigma_{V M}^{\max } \approx 79 \mathrm{MPa}$ for $f=4 \%$ ). Since the allowable maximum von Mises stress is $\sigma_{a}=67,5 M P a, \sigma_{V M 2}^{\max }<\sigma_{a}$, which makes that stress objective is met with $f=4 \%$.

It must be pointed out that, at this point of our research, this strategy for stress-based SIMP optimization is rather intuitive since increasing $f$ results in increasing material volume in the reconstructed structure which results in reducing stress. However, this result opens the path for further research work towards fully automating stress-based optimization.
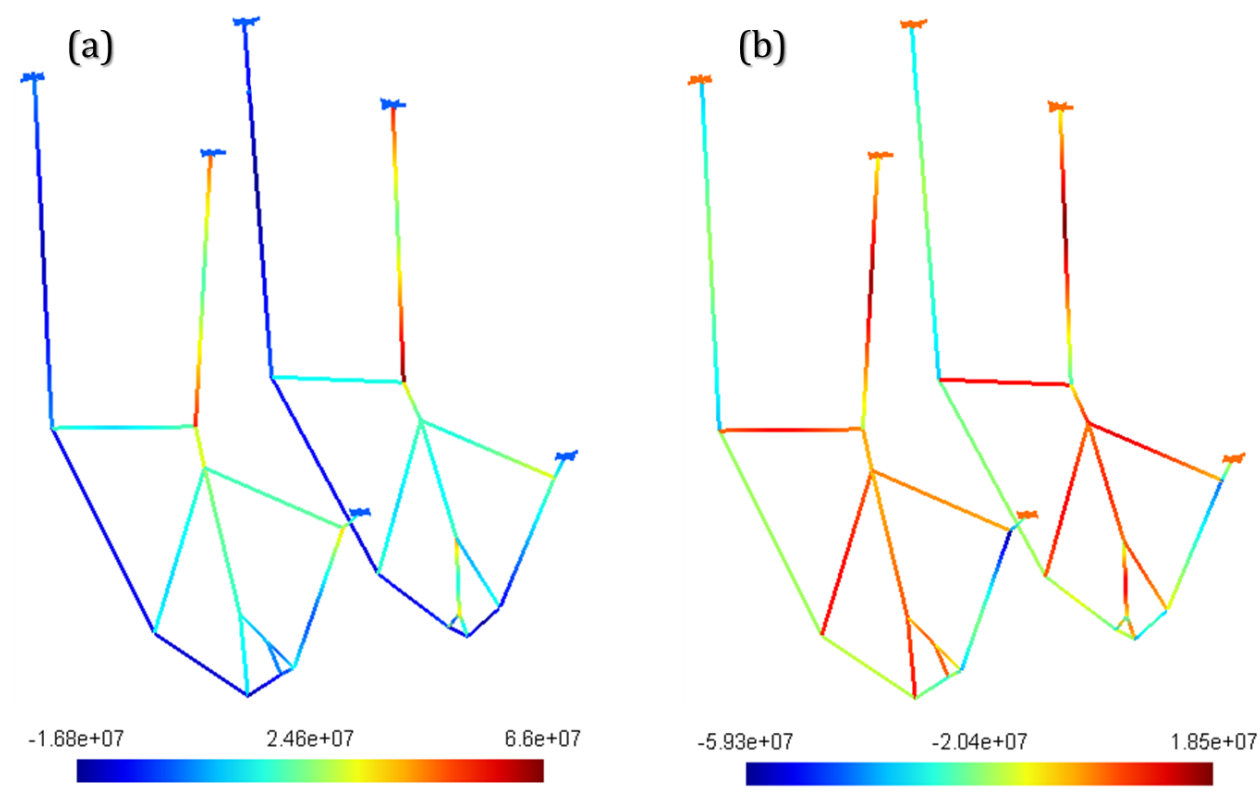

Figure 29: L-bracket with $f=5 \%$. Distribution of (a) maximum $\sigma_{x}$ stress (b) minimum $\sigma_{x}$ stress.

\section{Conclusion}

In this paper, we have proposed an automatic process for reconstructing straight beam structures from topology optimization (TO) results that tends to beam-like structures. The optimization process is based on the SIMP method and the model reconstruction strategy includes a curve skeletonization process. Curve skeletons are approximated as straight beams with basic geometric circular cross sections. A mixed-dimensional beam-solid finite element analysis (FEA) is conducted to validate the optimized model topology and geometry. This mixeddimensional FEA features mini-beams elements to couple beam and 3D solid elements. With our approach, we are able to automatically reconstruct rough optimized results into CAD designs, which are then easier to modify, to manipulate, and to manufacture. The proposed approach offers capabilities for fully integration of a TO method in the overall structural design procedure in 3D. Even if promising and applicable regardless the TO method used, the current method is, at this point of our research, limited to beam-like structures and to constant cross section beams. Therefore, reconstructing 3D structures made of standard beams with various cross section 
shapes is a natural potential extension of this work. Using alternative curve skeletonization techniques such as those suggested in [27] could also be investigated to improve efficiency of the approach. Also, further processing could be foreseen to improve the consistency of reconstruction results. As suggested in the result shown in Figure 30a (taken from Figure 15c), constraints could be applied in the process to improve consistency of the optimized structure and automatically generate the result shown in Figure 30b. For this example, 3 pairs of joints are merged and 2 groups of nearly aligned beams are merged into 2 straight beams. In this case, this is performed manually and automating this type of operations represents a very interesting extension of the work presented in this paper. The application of the reconstruction approach presented in this paper to other 3D TO methods, such as ESO, BESO, level sets, etc., or to adapted TO as proposed in [33] is another potential extension of this work.
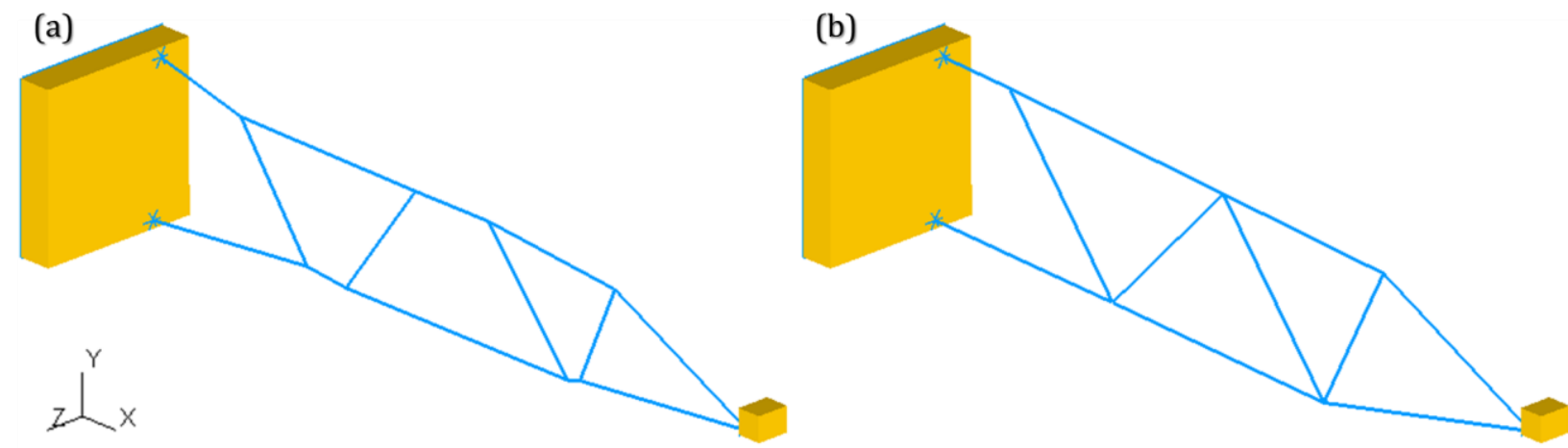

Figure 30 : Cantilever normalized skeleton (a) before and (b) after improvement.

\section{Acknowledgement}

This study was carried out as part of a project supported by research funding from the Natural Sciences and Engineering Research Council of Canada (NSERC) and UQTR foundation.

\section{References}

[1] Deaton JD, Grandhi RV. A survey of structural and multidisciplinary continuum topology optimization: Post 2000. Structural and Multidisciplinary Optimization. 2014;49(1):1-38.

[2] Allaire G, Belhachmi Z, Jouve F. The homogenization method for topology and shape optimization. Single and multiple loads case. Revue Europeenne des Elements. 1996;5(5-6):64972.

[3] Van Dijk NP, Maute K, Langelaar M, van Keulen F. Level-set methods for structural topology optimization: a review. Structural and Multidisciplinary Optimization. 2013;48(3):43772.

[4] Xie YM, Steven GP. Optimal design of multiple load case structures using an evolutionary procedure. Engineering Computations. 1994;11(4):295-302.

[5] Bendsoe MP, Sigmund O. Topology optimization - Theory,Methods and Applications. 2nd ed. Berlin: Springer; 2003. 
[6] He Q, Kang Z, Wang Y. A topology optimization method for geometrically nonlinear structures with meshless analysis and independent density field interpolation. Computational Mechanics. 2014;54(3):629-44.

[7] Liu J, Ma Y. A survey of manufacturing oriented topology optimization methods. Advances in Engineering Software. 2016;100:161-75.

[8] Youn S-K, Park S-H. A study on the shape extraction process in the structural topology optimization using homogenized material. Computers \& Structures. 1997 1997/02/01;62(3):52738.

[9] Hsu M-H, Hsu Y-L. Interpreting three-dimensional structural topology optimization results. Computers \& Structures. 2005;83(4-5):327-37.

[10] Koguchi A, Kikuchi N. A surface reconstruction algorithm for topology optimization. Engineering with Computers. 2006;22(1):1-10.

[11] Tang P-S, Chang K-H. Integration of topology and shape optimization for design of structural components. Structural and Multidisciplinary Optimization. 2001;22:65-82.

[12] Chang K-H, Tang P-S. Integration of design and manufacturing for structural shape optimization. Advances in Engineering Software. 2001;32:555-67.

[13] Papalambros PY, Chirehdast M. An integrated environment for structural configuration design. Journal of Engineering Design. 1990;1(1):73-96.

[14] Chirehdast M, Gea H-C, Kikuchi N, Papalambros P. Structural configuration examples of an integrated optimal design process. Journal of Mechanical Design. 1994;116(4):997-1004.

[15] Bremicker M, Chirehdast M, Kikuchi N, Papalambros P. Integrated topology and shape optimization in structural design. Journal of Structural Mechanics. 1991;19(4):551-87.

[16] Lin C-Y, Chao L-S. Automated image interpretation for integrated topology and shape optimization. Structural and Multidisciplinary Optimization. 2000;20(2):125-37.

[17] Chou Y-H, Lin C-Y. Improved image interpreting and modeling technique for automated structural optimization system. Structural and Multidisciplinary Optimization. 2010;40(1-6):21526.

[18] Larsen S, Jensen CG. Converting topology optimization results into parametric CAD models. Computer-Aided Design and Applications. 2009;6(3):407-18.

[19] Tagliasacchi A, Delame T, Spagnuolo M, Amenta N, Telea A. 3D skeletons: A state-of-theart report. Computer Graphics Forum. 2016;35(2):573-97.

[20] Blum H. A Transformation for Extracting New Descriptions of Shape. Models for the Perception of Speech and Visual Form, MIT Press, Boston, USA 1967:362-80.

[21] Sherbrooke EC, Patrikalakis NM, Wolter FE. Differential and topological properties of medial axis transforms. Graphical Models and Image Processing. 1996;58(6):574-92.

[22] Foskey M, Lin MC, Manocha D. Efficient computation of a simplified medial axis. Journal of Computing and Information Science in Engineering. 2003;3(4):274-84.

[23] Cornea ND, Demirci MF, Silver D, Dickinson S, Kantor P. 3D object retrieval using manyto-many matching of curve skeletons. International Conference on Shape Modeling and Applications 2005 (SMI'05); 2005: IEEE; 2005. p. 366-71.

[24] Tagliasacchi A, Zhang H, Cohen-Or D. Curve skeleton extraction from incomplete point cloud. ACM Transactions on Graphics (TOG); 2009: ACM; 2009. p. 71.

[25] Culver T, Keyser J, Manocha D. Exact computation of the medial axis of a polyhedron. Computer Aided Geometric Design. 2004;21(1):65-98.

[26] Svensson S, Nyström I, di Baja GS. Curve skeletonization of surface-like objects in 3D images guided by voxel classification. Pattern Recognition Letters. 2002;23(12):1419-26. 
[27] Au OK-C, Tai C-L, Chu H-K, Cohen-Or D, Lee T-Y. Skeleton extraction by mesh contraction. ACM Transactions on Graphics (TOG). 2008;27(3):44.

[28] Tagliasacchi A, Alhashim I, Olson M, Zhang H. Mean curvature skeletons. Computer Graphics Forum; 2012: Wiley Online Library; 2012. p. 1735-44.

[29] Díaz AR, Belding B. On optimum truss layout by a homogenization method. Journal of Mechanical Design. 1993;115(3):367-73.

[30] Cuillière JC, Francois V. Integration of CAD, FEA and topology optimization through a unified topological model. Computer-Aided Design and Applications. 2014 //;1(5):1-15.

[31] Sigmund O. A 99 line topology optimization code written in Matlab. Structural and Multidisciplinary Optimization. 2001;21:120-7.

[32] Wang Y, Kang Z, He Q. Adaptive topology optimization with independent error control for separated displacement and density fields. Computers and Structures. 2014;135:50-61.

[33] Nana A, Cuillière J-C, Francois V. Towards adaptive topology optimization. Advances in Engineering Software. 2016 10//;100:290-307.

[34] Code Aster. [cited; Available from: http://www.code-aster.org/

[35] Geuzaine C, Remacle J-F. Gmsh: a three-dimensional finite element mesh generator with built-in pre- and post-processing facilities. International Journal for Numerical Methods in Engineering. 2009;79(11):1309-31.

[36] Chen C-Y, Cheng K-Y. A direction-oriented sharpness dependent filter for 3D polygon meshes. Computers \& Graphics. 2008;32(2):129-40.

[37] Taubin G. A signal processing approach to fair surface design. Proceedings of SIGGRAPH '95; 1995 August 6-11; Los Angeles: ACM Press; 1995. p. 351-8.

[38] Clark B, Ray N, Jiao X. Surface mesh optimization, and untangling with high-order accurancy. International Meshing Roundtable; 2012; San-José; 2012.

[39] Cao J, Tagliasacchi A, Olson M, Zhang H, Su Z. Point cloud skeletons via laplacian based contraction. Shape Modeling International Conference (SMI), 2010; 2010: IEEE; 2010. p. 18797.

[40] Bournival S, Cuillière J-C, François V. A mesh-geometry based method for coupling 1D and 3D elements. Advances in Engineering Software. 2010;41(6):838-58.

[41] Cuillière J-C, François V, Drouet J-M. Automatic mesh generation and transformation for topology optimization methods. Computer-Aided Design. 2013;45(12):1489-506.

[42] Sobiecki A, Yasan HC, Jalba AC, Telea AC. Qualitative comparison of contraction-based curve skeletonization methods. International Symposium on Mathematical Morphology and Its Applications to Signal and Image Processing; 2013: Springer; 2013. p. 425-39. 